\title{
Über die syntaktische Position der Satztopiks im Deutschen*
}

\author{
Werner Frey \\ Zentrum für Allgemeine Sprachwissenschaft, Berlin \\ frey@zas.gwz-berlin.de
}

\section{Inhalt}

Das Papier argumentiert anhand einer Reihe von Phänomenen für die Existenz einer ausgezeichneten Topikdomäne im Mittelfeld des deutschen Satzes. Deutsch ist somit Diskurs-konfigurational hinsichtlich Topiks. Die Beobachtung erlaubt die Beantwortung einiger grundlegender Fragen wie die nach der möglichen Anzahl von Satztopiks, nach der Möglichkeit von Satztopiks in eingebetteten Sätzen oder nach dem Zusammenhang von Scrambling und Topikstatus. Die These, die 'starke' Interpretation einer indefiniten Phrase impliziere deren Topikstatus, wird zurückgewiesen. Syntaktische Eigenschaften der Topik-Voranstellung im Mittelfeld werden herausgearbeitet und ihre Implikationen für die Theoriebildung werden erörtert.

\section{Das Satztopik}

Die berühmte Charakterisierung des Konzepts 'Satztopik'1 durch Hockett (1958:201) lautet:

(1) "the most general characteristic of predicative constructions is suggested by the terms 'topic' and 'comment' for their ICs: the speaker announces a topic and then says something about it"

Die folgenden Beispiele können diese Beschreibung illustrieren²:

(2) a. /Peter forderte Maria zum ITANzen auf

b. Peter forderte Maria zum \TANzen auf

c. Die PolilZEI kommt

(2a) wird so verstanden, daß einem informationsstrukturell hervorgehobenen Referenten eine bestimmte Eigenschaft zugewiesen wird. Dabei muß dieser Referent nicht als im Kontrast zu anderen stehend aufgefaßt werden. Der Referent des Ausdrucks 'Peter' ist der Ausgangspunkt des Satzes. Über ihn wird prädiziert. ${ }^{3}$ Der Ausdruck 'Peter' in (2a)

" Für wertvolle Hinweise danke ich Claudia Maienborn, Karin Pittner, Marga Reis, Michael Grabski und Chris Wilder.

1 Der Terminus 'Satztopik' wird in der Literatur mehrdeutig verwandt. Mit ihm wird sowohl eine Konstituente im Satz bezeichnet, als auch auf ein Objekt der Welt referiert. Im Zitat von Hockett treten beide Verwendungsweisen sogar in einer Äußerung auf. Im folgenden Text verweist der Terminus auf eine Satzkonstituente.

2 Großbuchstaben markieren betonte Silben: /: steigend, ।: fallend.

3 (2a) muß daher unterscheiden werden von der sog. I-Topikalisierung, wie man sie im folgenden Beispiel findet: 
piks benannt werden. Reinhart (1981) vergleicht dies mit dem Ordnungsprinzip des thematischen Katalogs einer Bibliothek. Ein Topik funktioniert hiernach wie ein Thema, dem Einträge zugeordnet werden.

Der Aufsatz ist wie folgt gegliedert: Im Abschnitt 2 wird die Frage nach der syntaktischen Auszeichnung der Topiks im Deutschen aufgeworfen. Es wird dafür argumentiert, daß im deutschen Mittelfeld eine Domäne für die strukturelle Auszeichnung der Topiks existiert. Abschnitt 3 zieht Folgerungen aus diesem Befund, u.a. wird die Frage diskutiert, für welche der beiden Explikationen (4i) oder (ii) die strukturell ausgezeichneten Topiks des Deutschen sprechen. Abschnitt 4 wirft einen Blick auf Pronomina. In Abschnitt 5 wird die These des Zusammenhangs der sog. starken Interpretation indefiniter NPs und deren Topikstatus zuückgewiesen. In Abschitt 6 wird diskutiert, welche Elemente neben NPs als Satztopiks möglich sind. Abschnitt 7 enthält eine Auseindersetzung mit alternativen Vorschlägen zur Syntax der Topiks im Deutschen. In Abschnitt 8 wird die Topikauszeichnug mit sonstiger Mittelfeldumstellung und mit der Vorfeldbesetzung verglichen. Schließlich werden in Abschnitt 9 Überlegungen angestellt, wie sich die Mittelfeldauszeichnung der Topiks in die Syntax des deutschen Satzes eingliedern läßt.

\section{Der Topikbereich im Mittelfeld des deutschen Satzes}

Die meisten Autoren gehen davon aus, daß es im Deutschen keine speziell für Topiks reservierte Position gibt (z.B. Molnár (1991, 1998), Lambrecht (1994), Vallduví \& Engdahl (1996), Jacobs (1999)). Bei Sätzen ohne Herausstellung ist der Blick auf das Vorfeld gerichtet. Molnár $(1991,1998)$ nimmt an, daß Topiks im Deutschen notwendigerweise im Vorfeld plaziert werden. Die obligatorische Initialstellung wird aber nicht nachgewiesen. Auch Jacobs (1999) nennt als eine prototypische Eigenschaft des Topiks, daß es auf der S-Struktur von keinem anderen Argument des Satzes k-kommandiert wird. ${ }^{4}$ Da man aber auch feststellt, daß im Vorfeld nicht nur Topiks positioniert werden, ergibt sich, daß Deutsch bezüglich Topiks nicht Diskurs-konfigurational ist ${ }^{5}$.

Im vorliegenden Papier wird für eine andere These argumentiert. Für diese wird die Position der Satzadverbiale eine wichtige Rolle spielen. Daher seien sie zunächst charakterisiert.

Die Grundzüge (1981) definieren die Satzadverbiale (= Modalwörter) als jene Adverbiale, die eine Einschätzung des im Satz beschriebenen Sachverhalts durch den Sprecher bezeichnen. Diese Verwendungsweise des Terminus 'Satzadverbial' wird hier ebenfalls zugrundegelegt. Daraus folgt, daß z.B. Temporal- oder Kausaladverbiale nicht zu den Satzadverbialen gezählt werden. Beispiele für Satzadverbiale sind: Evaluative Adverbiale ("erstaunlicherweise'), Evidenzadverbiale ('offensichtlich'), Epistemische Adverbiale ("wahrscheinlich").

Satzadverbiale besitzen neben ihrer kanonischen Verwendung mit Satzbezug auch eine Verwendung als fokussierende Elemente. Sie nehmen dabei wie in (5a) Bezug auf eine

4 Die Annahme einer obligatorischen Initalstellung hat eine lange Tradition: Halliday (1967) definiert das Satztopik als den ersten Ausdruck in einem Satz. Aber bereits Reinhart (1981) weist diesen Ansatz als für das Englische nicht haltbar zurück.

5 Da Topiks im Deutschen auch nicht intonatorisch markiert zu werden brauchen (s. (2b)), scheint Deutsch hiernach zu jenen Sprachen zu gehören, auf die sich T. Reinhart $(1995,105)$ bezieht: "One of the factors that make topics a harder subject for research than, say, foci, is that in most languages they are not marked either intonationally or syntactically." 
einzelne Konstituente des Satzes. In dieser Verwendung können sie sogar gemeinsam mit ihrem Bezugselement im Vorfeld auftreten, s. (5b).

(5) a. Jemand hat die Frau vermutlich im PARK bestohlen

b. Unglücklicherweise dem OTto hat sich Maria anvertraut

In dieser Verwendungsweise der Satzadverbiale präsupponiert der Satz jene Proposition, die aus der Proposition ohne Satzadverbial dadurch entsteht, daß der fokussierte Teil durch eine existentiell gebundene Variable ersetzt wird. Diese Verwendung der Satzadverbiale spielt im folgenden keine Rolle. Die Satzadverbiale der Beispiele sollen stets mit Satzbezug verstanden werden. In der Verwendung mit Satzbezug induzieren die Satzadverbiale keine Präsupposition.

Nach Frey \& Pittner (1998) haben die verschiedenen Adverbialklassen unterschiedliche Grundpositionen im deutschen Mittelfeld des deutschen Satzes. Die Satzadverbiale sind hiernach sehr hoch in der Struktur positioniert. Ihre Grundposition ist höher als die Grundpositionen der Argumente und der anderen Adverbialklassen.

Ich möchte nun die folgende zentrale These aufstellen, die einen Hinweis aus Frey \& Pittner (1998) und Pittner (1999) aufgreift:

(6) Direkt den Satzadverbialen vorangehend gibt es im Mittelfeld des deutschen Satzes einen ausgezeichneten Bereich für Topiks: Alle topikalen Phrasen des Mittelfelds und nur diese werden in diesem Bereich positioniert.

Wir nennen im folgenden diesen Bereich den Topikbereich des Satzes. Die These in (6) soll nun durch eine Reihe empirischer Beobachtungen und Tests belegt werden.

(I) 'aboutness'-Kontext

Betrachten wir das folgende Beispiel:

(7) Ich erzähl dir mal was von Otto.

a. Nächstes Jahr wird Otto wahrscheinlich seine Kollegin heiraten

b. \#Nächstes Jahr wird wahrscheinlich Otto seine Kollegin heiraten

(7b) ist im gegebenen Kontext nicht möglich. Dies wird durch (6) erfaßt. Durch den Vorsatz wird 'Otto' als Topik der folgenden Aussage festgesetzt. Nach (6) muß aber ein Satztopik vor einem Satzadverbial auftreten.

Es ist wichtig zu beachten, daß es nicht der Definitheit-Status einer Phrase ist, welcher für die Voranstellung verantwortlich ist. Ein definites Subjekt muß nicht vor dem Satzadverbial stehen:

(8) Heute wird wahrscheinlich Fredi Bobic im Dortmunder Sturm spielen

Erst dadurch, daß das Denotat einer Phrase zum Topik gemacht wird, wird die Voranstellung der Phrase erzwungen.

(7) zeigt, daß ein topikalisches Subjekt im Mittelfeld vor einem Satzadverbial auftreten muß. Dies gilt genauso für Objekte: 
(9) Ich erzähl dir mal was von Otto.

a. Nächsten Monat wird den Otto erfreulicherweise die Botschafterin von Norwegen heiraten

b. \#Nächsten Monat wird erfreulicherweise den Otto die Botschafterin von Norwegen heiraten

(9a) zeigt darïber hinaus, daß ein Objekt auch bei Präsenz eines definiten Subjekts durchaus (alleiniges) Topik sein kann.

(II) nicht-referentielle Ausdrücke

Das nächste Datum betrifft quantifizierte Elemente wie 'keiner' oder 'fast jeder'. Sie kommen aufgrund ihrer Semantik nicht als Topik in Frage. Topiks als Repräsentanten der Kategorie des 'Worüber' müssen eindeutig identifizierbare Diskursreferenten bereitstellen, über die prädiziert wird. In der Metaphorik der Adressen von Reinhart müssen Topiks eindeutige Adressen abgeben, denen Information zugeordnet werden kann. Dies können Elemente wie 'keiner' oder 'fast jeder' nicht. Aber auch nach der 'familiarity'Konzeption sind dies offensichtlich keine möglichen Topiks.

Als Elemente, die nicht Topik-fähig sind, können sie nicht im Topikbereich des Satzes auftreten:

(10) a. *Während des Vortrags hat keiner anscheinend geschlafen

b. ??Im Stadion hat fast jeder wahrscheinlich das Handspiel gesehen

(III) Das Bezugselement kataphorischer Pronomen

Nach Reinhart (1981, 1995) (aufbauend auf Kuno (1972)) ist ein kataphorisches Pronomen nur mit Bezug auf ein Topik möglich.

(11) a. Da er, gut vorbereitet ist, wird Fred, wahrscheinlich einen interessanten Vortrag halten

b. *Da er, gut vorbereitet ist, wird wahrscheinlich Fred, einen interessanten Vortrag halten

c. Sein $_{1}$ Vater wird dem Otto 1 glücklicherweise das Auto ausleihen

d. $\quad$ Sein $_{1}$ Vater wird glücklicherweise dem Otto ${ }_{1}$ das Auto ausleihen

e. Da er betrunken ist, wird den Felix wahrscheinlich der Max beschimpfen

f. Da er betrunken ist, wird der Max wahrscheinlich den Felix beschimpfen

g. Da er betrunken ist, wird der Max den Felix wahrscheinlich beschimpfen

Nur wenn das potentielle Bezugselement nach (6) durch die syntaktische Struktur als Topik ausgewiesen wird, ist das kataphorische Pronomen möglich, s. (11a, c). ${ }^{6}$ Man beachte, daß sich damit auch die Interpretationen für (11e-g) erklären. (11e) wird so verstanden, daß Felix betrunken ist, (f) hingegen so, daß dies auf Max zutrifft. Im Beispiel (11g) sind beide Interpretationen möglich.

6 Diese Daten haben nichts mit Rekonstruktion der Phrasen im Vorfeld für Bindungszwecke zu tun. Es handelt sich in diesen Beispielen um Koreferenz, nicht um Bindung. Bindung der Pronomen in der Vorfeldphrase wäre in allen Fällen in (11) nicht möglich. 


\section{(IV) 'Topik-sensitive' Demonstrativpronomen}

Ein weiteres Testinstrument für den Topikstatus eines Elementes stellen im Holländischen nach Reinhart (1995) bestimmte Demonstrativpronomen dar. Diese meiden Antezedenten mit einer hohen Zugänglichkeit (Reinhart $(1995,102))$. Topiks haben eine hohe Zugänglichkeit.

Die folgenden Daten des Deutschen sind allerdings nur von eingeschränkter Aussagekraft. Es gibt Sprecher, die generell das Pronomen 'derselbige' als merkwürdig empfinden. Für mich jedoch besteht ein deutlicher Kontrast zwischen den folgenden Beispielen:

(12) a. Heute hat Hans ${ }_{1}$ erfreulicherweise einen Job bekommen. ${ }^{*}$ Derselbige 1 hat es wirklich verdient.

b. Heute hat erfreulicherweise Hans, einen Job bekommen. Derselbige ${ }_{1}$ hat es wirklich verdient.

\section{(V) Phrasen mit steigendem Akzent}

Das Beispiel (2a) hat gezeigt, daß es eine Akzentuierung einer vorangehenden Phrase gibt, dic in einem Satz mit einem zweiten prominenten Akzent zu ihrer Auszeichnung als Satzgegenstand führt. Dabei liegt keine kontrastierende Lesart vor. Betrachten wir nun derart betonte Phrasen im Mittelfeld:

(13) a. Heute abend wird /PEter vermutlich ins Schloßcafé zum \TANzen gehen

b. Heute abend wird vermutlich /PEter ins Schloßcafé zum ITANzen gehen

Man stellt fest, daß in (13a) der Referent von 'Peter' als Gegenstand der Aussage ausgezeichnet wird. (13b) hingegen kann nur so verstanden werden, daß Peter in Kontrast zu anderen Individuen gesetzt wird. Hier liegt nicht die neutrale Auszeichnung als Satztopik vor.

(VI) Thetische Sätze, Präsentativkonstruktionen

Thetische Sätze gelten als Paradebeispiele einer topikfreien Konstruktion?:

(14) Was passiert gerade?
a. Gerade wird vermutlich der neue PräsilDENT gewählt
b. *Gerade wird der neue PräsilDENT vermutlich gewählt
c. Im Moment kommt hoffentlich die PoliVEI
d. *Im Moment kommt die PolilZEI hoffentlich

(14) zeigt, daß das Subjekt eines thetischen Satzes nicht in dem Bereich auftreten kann, der nach (6) den Topikbereich darstellt.

Von den sog. Präsentativkonstruktionen wird angenommen, daß sie kein topikales Subjekt aufweisen (Kuno (1972)). Beispiele für Präsentativkonstruktionen im Deutschen sind:

7 Als thetisch sollen hier die Sätze bezeichnet werden, bei denen auf das Subjekt ein fallender Hauptakzent fällt, wobei der gesamte Satz focal ist. In einem sochen Fall wird durch die formale Markierung erzwungen, daß ein Satz ein Ereignis ungegliedert, d.h. Topik-frei, präsentiert (vgl. Drubig (1992)). Teilweise wird in der Literatur allerdings 'thetischer Satz' generell als synonym mit 'beliebiger Satz ohne Topik' verwandt. 
(15) a. Es spielt Erwin Lehn die ganze Nacht hindurch ('V2-Satz mit Expletiv im Vorfeld')

b. Kam Hans zur Tür herein und ...

('Narrativer V1-Satz')

Man beachte, daß in dieser Konstruktion durchaus definite, referentielle Subjekte vorkommen können. Aber diese sind eben nach Kuno keine möglichen Satztopiks. Wir erwarten daher, daß die Subjekte von (15) nicht vor Satzadverbialen auftreten können. Diese Erwartung bestätigt sich:

(16) a. *Es spielt Erwin Lehn erfreulicherweise die ganze Nacht hindurch

b. Es spielt erfreulicherweise Erwin Lehn die ganze Nacht hindurch

c. *Kam Hans überraschenderweise zur Tür herein und ...

d. Kam überraschenderweise Hans zur Tür herein und ...

Interessanterweise können in einer Präsentativkonstruktion zwar Subjekte nicht Topik sein und deshalb nicht im Topikbereich auftreten, andere Elemente sind jedoch als Topik möglich und daher im Topikberreich situierbar"

(17) a. Es spielt für sie erfreulicherweise Erwin Lehn die ganze Nacht hindurch

b. Es hat den Otto leider jemand heftig beschimpft

(VII) Topiksensitive Adverbien

Es gibt Adverbien, deren Bezugselement bei Normalbetonung des Satzes Topikstatus aufweist. Zu ihnen gehört 'jedenfalls':

(18) a. weil [Peter jedenfalls] zum Glück morgen mithelfen wird

b. *weil zum Glück [Peter jedenfalls] morgen mithelfen wird

c. Maria wird [dem Peter jedenfalls] zum Glück die Unterlagen geben

d. *Maria wird zum Glück [dem Peter jedenfalls] die Unterlagen geben

(18) zeigt, daß unabhängig von der grammatischen Funktion eine durch das Adverb erweiterte NP vor dem Satzadverbial stehen muß ${ }^{9}$.

8 Dies gilt auch für andere Präsentativkonstruktionen;

(i) a. Hier wird (dem Gast) erfreulicherweise frisches Gemüse angeboten

b. *Hier wird frisches Gemüse erfreulicherweise (dem Gast) angeboten

9 Diesen Test für die Behauptung (6) verdanke ich Marga Reis (p.M.). Ein entsprechendes Verhalten zeigt auch 'aber', wenn es mit einer NP assoziert ist:

(i) a. weil [Peter aber] leider nicht mithelfen wird

b. *weil leider [Peter aber] nicht mithelfen wird

Unter Kontrastfokus der assozierten NP ist es jedoch möglich, die Phrasen nach einem Satzadverbial zu stellen:

(ii) a. weil glücklicherweise [PETer jedenfalls] morgen mithelfen wird (wenn auch nicht OTto)

b. weil morgen leider [PETer aber] nicht mithelfen wird (jedoch wenigstens OTto) 
(VIII) Die Konstruktion mit einer gespaltenen NP

In der Regel werden bei gespaltenenen NPs Beispiele betrachtet, in denen der Teil, der das Nomen enthält, im Vorfeld auftritt. Man hat dabei die Intuition, daß die Vorfeldkonstituente Topikstatus hat ${ }^{10}$ :

(19) Volvos 1 hat er nur blaue $t_{1}$ gekauft

Aber die Spaltung kann auch im Mittelfeld erfolgen. Wir können dabei die These (6) überprüfen. Die Beispiele sind eindeutig:

(20) a. weil Hans Volvos $_{1}$ leider nur blaue $t_{1}$ gekauft hat

b. * weil Hans leider Volvos $_{1}$ nur blaue $\mathrm{t}_{1}$ gekauft hat

c. *weil Hans Volvos $_{1}$ nur blaue $\mathrm{t}_{1}$ leider gekauft hat

d. Otto wird Bücher ${ }_{1}$ wahrscheinlich keine $t_{1}$ verschenken

e. $*$ Otto wird wahrscheinlich Bücher keine $_{1}$ verschenken

Der Teil mit dem Nomen muß im Topikbereich auftreten (s. (20b,e)), der Rest der aufgespaltenen NP darf dort nicht stehen (s. (20c)).

Zusammenfassend können wir feststellen, daß die Daten in (I)-(VIII) die These (6) belegen: Es gibt im Mittelfeld des deutschen Satzes eine syntaktische Auszeichnung der Topiks. Die Annahme, Topiks müßten im Deutschen notwendigerweise im Vorfeld plaziert werden, ist nicht richtig.

Deutsch gehört somit (hinsichtlich Topiks) zu den Diskurs-konfigurationalen Sprachen in der Terminologie von Kiss (1995): Es gibt eine syntaktische Position, in der Topiks ausgezeichnet werden.

\section{Folgerungen aus (6)}

In diesem Abschnitt sollen einige Konsequenzen erörtert werden, die sich aus (6) ergeben. Dabei wird mit den Punkten (III)-(VII) auch zu Fragen Stellung bezogen, die in der Topikforschung seit langem kontrovers diskutiert werden.

(I) Es sind die Satzadverbiale und keine anderen Adverbialtypen, die die Grenze zum Topikbereich bilden.

Man vergleiche (10) mit:

(21) a. weil keiner heute zu spät gekommen ist

b. da fast jeder in diesem Zimmer gearbeitet hat

Eine nicht-referentielle Phrase kann einem Temporaladverbial wie in (21a) oder einem Lokaladverbial wie in (21b) vorangehen. Dies zeigt, daß vor diesen Adverbialen nicht nur Topiks stehen.

Nach Frey \& Pittner (1998) gehören Temporaladverbiale zu der Klasse von Adverbialen, die zwar unterhalb der Satzadverbiale aber ansonsten sehr weit oben in der Struktur positioniert sind. Temporaladverbiale werden hiernach z.B. strukturell höher

${ }^{10}$ Auf die Relevanz der gespaltenenen NPs für Fragen der Topikalität hat mich Karin Pittner aufmerksam gemacht. 
basisgeneriert als Lokaladverbiale. Es genügt demnach, bei der Anwendung weiterer Tests die Satzadverbiale mit den Temporaladverbialen zu vergleichen. Die anderen Tests aus Abschnitt 2 führen nun ebenfalls zum Resultat, daß eine Phrase, die einem Temporaladverbial vorangeht, dadurch noch nicht als Topik ausgezeichnet wird. Für Test (VII) zeigen dies bereits die Beispiele (18b, d). Für Test (VI) aus Abschnitt 2 vergleiche man den folgenden Satz mit (16a):

(22) Es spielt Erwin Lehn heute abend für Sie

Auch Test (III) z.B. führt zum selben Ergebnis:

(23) a. * Sein $_{1}$ Chef hat leider den Otto ${ }_{1}$ heute beleidigt

b. Sein ${ }_{1}$ Chef hat den $\mathrm{Otto}_{1}$ leider heute beleidigt

Beobachtungen wie diese unterstreichen auch die Wichtigkeit der systematischen Unterscheidung der verschiedenen Adverbialklassen.

(II) Ob im Vorfeld ein Topik steht oder nicht, ist unabhängig von der TopikAuszeichnung im Mittelfeld.

Wie allgemein angenommen, kann im Vorfeld ein Topik positioniert sein ${ }^{11}$, aber es muß dort kein Topik stehen. Letzteres erkennt man bereits daran, daß nicht-referentielle Ausdrücke, welche ja ungeeignet sind als Topiks, Vorfeld-fähig sind. Ob im Vorfeld ein Topik steht oder nicht, ist, wie man sich leicht klar macht, unabhängig von einer etwaigen Topik-Auszeichnung im Mittelfeld.

\section{(III) Es kann mehrere Satztopiks im einfachen Satz geben.}

Mehrere Konstituenten können einem Satzadverbial vorangehen. Dies ist etwa der Fall in (11g). Wenn mehrere Elemente im Topikbereich auftreten, können sie, wie erwartet, alle Bezugselemente kataphorischer Pronomen sein:

(24) Da er 1 sie 2 so liebt wird Hans 1 Maria 2 wahrscheinlich bald einen Antrag machen.

Einige Autoren vertreten allerdings die Ansicht, ein Satz könne maximal ein Satztopik aufweisen (Reinhart (1981, 1995), Haftka (1995), Zimmermann (1999), Molnár (1991, 1998), Jacobs (1999) für das Deutsche), dies geschieht jedoch oft ohne weitere Diskussion. Innerhalb der aboutness-Ansätze scheint die stillschweigende Annahme zu sein, daß sich die Relation des 'Worüber' nur auf ein Element beziehen kann. Dies aber ist nicht selbstverständlich. Es ist plausibel, anzunehmen, daß eine Proposition durchaus über mehrere Elemente ausgesagt werden kann. Um Reinharts Vergleich mit dem thematischen Katalog einer Bibliothek aufzunehmen: Ein Buch kann ebenfalls mehreren Themen zugeordnet werden. Man beachte, daß z.B. Rizzi (1997:290) für das Italienische, Lasnik \& Saito (1992:78) für das Englische oder É. Kiss (1995a:211) für das Ungarische die Möglichkeit mehrerer Topiks pro Satz vorsehen. Für das Türkische ist es ebenfalls eine Standardannahme, daß mehrere Elemente im einfachen Satz Topiks sein können (Kornfilt (1997:205)).

11 Dies erkennt man z.B. an den folgenden Beipielen mit den Anwendungen der Tests (III), (V) und (VII):

(i) a. $\mathrm{Er}_{1}$ ist gut vorbeitet. Fred ${ }_{1}$ wird daher einen interessanten Vortrag halten.

b. /Peter wird heute ins Schloßcafé zum ITANzen gehen

c. Peter jedenfalls wird morgen mithelfen 
(IV) Es gibt Satztopiks in eingebetteten Sätzen.

Im folgenden Beispiel wird nach (6) im Komplementsatz ein Satztopik ausgezeichnet ${ }^{12}$ :

(25) Maria denkt, daß Hans glücklicherweise den Auftrag bekommen wird

Auch in Infinitivsätzen sind Topiks möglich ${ }^{13}$ :

(26) a. Er hat angekündigt, Maria wahrscheinlich zu wählen

b. Er beabsichtigt, Volvos 1 in Zukunft nur blaue $t_{1}$ zu kaufen

Interessanterweise kann der Satztopikstatus einer Konstituente eines eingebetteten Satzes im Diskurs verfügbar sein:

(27) [A:] Erzähl mal etwas von Hans.

[B:] a. Maria denkt, daß Hans glücklicherweise den Auftrag bekommen wird

b. \#Maria denkt, daß glücklicherweise Hans den Auftrag bekommen wird

Ein Sprecher kann sich die durch Verben wie 'denken' charakterisierte propositionale Einstellung einer anderen Person implizit zu eigen machen. Dabei kann er, wie (27a) zeigt, der eingebetteten Proposition die für die Diskursebene adäquate Informationsgliederung zuweisen.

Andere Verben der propositionalen Einstellung erlauben dieses Verfahren nicht:

(28) [A:] Erzähl mal etwas von Hans.

[B:] \#Maria bedauert, daß Hans anscheinend den Auftrag bekommen wird

(29) [A:] Erzähl mal, was Maria über Hans denkt.

[B:] a. Maria bedauert, daß Hans anscheinend den Auftrag bekommen wird

b. \#Maria bedauert, daß anscheinend Hans den Auftrag bekommen wird

Bei Verben wie 'bedauern' dient der Gehalt und die Informationsgliederung des eingebetteten Satzes ausschließlich der Charakterisierung der propositionalen Einstellung des Matrixsubjektes.

\section{(V) Anaphorische definite Phrasen sind im Deutschen nicht generell Topiks.}

Definite Phrasen, die auf im Text bereits eingeführte Referenten bezogen werden, sind nicht per se Satztopiks. Man betrachte die Positionierung von den Vater bzw. die Dame jeweils im zweiten Satz:

(30) a. Hans wird den Eltern von Maria vorgestellt. Er hat gestern die Mutter getroffen und heute wird er wahrscheinlich den Vater kennenlernen.

b. Gestern hat Otto eine elegante Geschäftsfrau kennengelernt. Er wird hoffentlich die Dame wiedersehen.

12 Die Möglichkeit von Topiks in eingebetteten Sätzen wird auch für andere Sprachen vorgesehen. Lasnik \& Saito (1992:76) z.B. diskutieren folgendes Beispiel:

(i) I believe that this book $_{1}$, you should read $t_{1}$

Rizzi (1997:Fn. 15) gibt ein italienisches Beispiel mit einem Satztopik im eingebetteten Satz.

13 Zum Topikstatus des abgespalteten Nomens s. oben in Abchnitt 3 unter (VIII). 
Natürlich können diese Konstituenten auch Topiks sein:

(31) a. Hans wird den Eltern von Maria vorgestellt. Gestern hat er die Mutter getroffen und heute wird er den Vater wahrscheinlich kennenlernen.

b. Gestern hat Otto eine elegante Geschäftsfrau kennengelernt. Er wird die Dame hoffentlich wiedersehen.

Eine Theorie wie die von Jäger (1996), in welcher folgt, daß eine anaphorische definite NP notwendigerweise Topik sein muß, ist somit mit (6) nicht verträglich.

Die Daten in (30) zeigen, daß mit (6) ein 'familiarity'-Konzept von Satztopiks nicht vereinbar ist, welches annimmt, daß Topiks genau die Konstituenten des Satzes sind, die auf im Diskurs bereits eingeführte bzw. durch 'bridging' verfügbare Referenten verweisen. Wäre 'familiarity' des Referenten einer Konstituente eine hinreichende Bedingung für den Topikstatus der Konstituente, so müßten die anaphorischen Phrasen in (30) Topiks sein. $^{14}$

(VI) (6) spricht für das 'aboutness'-Konzept für Satztopiks.

Reinhart (1981) sieht diverse Schwierigkeiten, die sich mit dem in (4i) benannten 'familiarity'-Konzept für Satztopiks ergeben. Das folgende Beispiel illustriert einen ihrer Punkte.

(32) Der FC geht voller Hoffnung in die neue Saison. Karl /MAIer soll die nötigen ITOre schießen. Der neue Stürmer konnte vom Lokalrivalen abgeworben werden und gilt als großes Talent.

Im zweiten Satz wird 'Karl Maier' als Topik verstanden. Dem Rezipienten muß aber der Referent des Ausdrucks vorher nicht gegenwärtig sein. Dies wird durch den Folgesatz sogar explizit gemacht, der erst die einführenden Informationen gibt.

Entsprechendes kann mit den durch (6) charakterisierten 'Mittelfeldtopiks' illustriert werden. Der erste Satz eines Textes kann durchaus eine solche Topikphrase aufweisen. Ein Zeitungsartikel könnte z.B. wie folgt beginnen:

(33) Heute wird Karl /MAIer wahrscheinlich sein erstes ISPIEL bestreiten. Der neue Stürmer vom FC ...

Im Topikbereich des Mittelfeldes kann auch eine indefinite NP auftreten, wie (34a) zeigt:

(34) a. da Hans zwei Artikel erfreulicherweise beabsichtigt zu lesen

b. da Hans erfreulicherweise zwei Artikel beabsichtigt zu lesen

Eine indefinite NP kann demnach Topik sein. Sie wird dann notwendigerweise 'stark' interpretiert, d.h. im vorliegenden Fall spezifisch oder partitiv. (34a) hat nur die Lesart, bei der der Sprecher sich auf zwei bestimmte Artikel bezieht oder auf eine bestimmte Menge von Artikeln, zu denen die fraglichen zwei gehören. Man vergleiche dies mit (34b), in dem die indefinite NP nicht topikal ist. Dieses Beispiel hat eine Lesart, bei der

14 Man beachte, daß sogar thetische Sätze mit anaphorischen Konstituenten möglich sind. Das folgende Beispiel findet sich in Jörke (1997):

(i) [A:] Warum trägst du deine Uhr nicht?

[B:] Die BatterUE ist leer 
Hans zwei beliebige Kapitel lesen will. In dieser Lesart muß der Sprecher keinerlei Wissen bezüglich der Artikel besitzen.

Eine indefinite NP führt einen neuen Diskursreferenten ein. Der Referent einer indefiniten NP ist somit für den Hörer nicht 'familiar'. 'Stark' gebrauchte indefinite NPs sind aber als Topik geeignet, da sich der Sprecher bei Verwendung 'starker' Indefinita auf bestimmte Individuen bezieht. Diese Verankerung des Ausdrucks wird vom Hörer akkomodiert.

Da eine 'stark' interpretierte indefinite NP Topik-fähig ist, vermag sie eine Katapher zu lizenzieren. Dies zeigt (35a):

(35) a. Damit er 1 auf ein Fußballinternat gehen kann, will Maria einen jungen Spieler ${ }_{1}$ unterstützen

b. Maria will einen jungen Spieler $r_{1}$ unterstützen, damit $\mathrm{er}_{1}$ auf ein Fußballinternat gehen kann

Der Satz (35a) hat nur die Lesart, bei der Maria einen bestimmten Spieler unterstützen will. Man vergleiche hiermit (35b). Dieses Beispiel kann so verstanden werden, daß Maria irgendeinen beliebigen Spieler unterstützen möchte. ${ }^{15}$

Unsere Beobachtungen zeigen, daß sich (6) mit dem 'aboutness'-Konzept für Satztopiks verbindet und nicht mit dem 'familiarity'-Konzept. Zum einen wurde bezüglich der Beispiele (30) festgestellt, daß 'familiarity' keine hinreichende Bedingung für Topiks im Sinne von (6) sein könnte. Wollte man 'familiarity' wenigstens zur notwendigen Bedingung für Topiks erklären, dann zeigen Daten wie (33)-(35), daß das Konzept auf Sprecher-'familiarity' eingeengt werden müßte. Dies entspräche aber nicht dem gängigen Verständnis, welches 'familiarity' als Präsenz im 'common ground' von Sprecher und Hörer ansetzt.

Es ist damit klar, daß sich bei Annahme von (6) die Topik-Kommentar-Gliederung prinzipiell unterscheidet von der Hintergrund-Fokus-Gliederung. Mit der Informationsgliederung 'Hintergrund-Fokus' werden Sätze in einen bekannten, erwarteten Teil (Hintergrund) und einen informativen, neuen Teil (Fokus) aufgespalten. In einem Satz sind jene Konstituenten fokal, deren semantischer Wert sich nicht nach Anwendung bestimmter semantischer Operationen aus gegebenen Elementen ableiten läßt (Schwarzschild (1999)). Betrachten wir ein einfaches Beispiel:

(36) a. Was wird Maria heute wahrscheinlich mit Otto machen?

b. [Hintergr. Heute wird Maria wahrscheinlich Otto] [Fokus durch Kreuzberg führen]

15 Das Subjektpronomen im zweiten Satz von (35b) ist Topik (s. Abschnitt 4). Obwohl also der Antezedent in der fraglichen Interpretation nicht Topik ist, ist es doch das Pronomen, welches sich auf ihn bezieht. Entsprechendes findet man in folgendem Satz:

(i) Fast jeder Teilnehmer ${ }_{1}$ nimmt an, daß $\mathrm{er}_{1}$ wahrscheinlich gewinnen wird

Der Antezedent ist nicht Topik-fähig, das von ihm gebundene Pronomen ist aber (nach Abschnitt 4) ein Topik. Diese Beispiele zeigen, daß die Redeweise 'Topiks sind referentiell' etwas irreführend ist. Sie sollte ersetzt werden durch die Redeweise 'Topiks sind verankert'. Der semantische Wert eines Topiks muß eindeutig bestimmt sein. In einer Lesart von (35b) oder in (i) ist das Pronomen nicht referentiell. Das Pronomen wird aber verankert, da die Variable, die das Pronomen einführt, mit der Variablen, die der Quantor einführt, identifiziert wird. 
Der informative Teil von (36b) als Antwort auf (36a) besteht aus der Auskunft 'durch Kreuzberg führen'. Deshalb ist im gegebenen Kontext von (36b) die Konstituente, die das Verb und die PP dominiert, eine fokale Konstituente.

Eine mögliche Topik-Kommentar-Gliederung des Satzes ist die folgende:

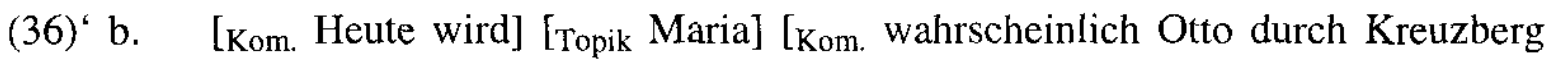
führen]

'Otto', 'heute', das Satzadverbial und die Tempusinformation gehören somit in diesem Beispiel zum Hintergrund ohne Topik zu sein.

Wegen der Unabhängigkeit der beiden Gliederungsprinzipien 'Topik-Kommentar' und 'Hintergrund-Fokus' ist es nicht erstaunlich, daß ein Topik Teil des Fokusbereiches sein kann ${ }^{16}$, s. (33) oder das folgende Beispiel:

(37) a. Was ist heute passiert?

b. Heute hat [Fokus [Topik der Studentenvertreter] leider heftig protestiert]

\section{(VII) Die I-Topikalisierung per se ist keine Topik-Kommentar-Konstruktion.}

Die sog. I-Topikalisierung zeichnet sich durch ein spezielles Intonationsmuster aus ('Wurzelkontour' nach Jacobs (1996)). Auf der ersten Akzentposition fällt der Ton zunächst etwas ab und steigt dann an. Auf der zweiten Akzentposition fällt er. Die erste Akzentposition befindet sich häufig im Vorfeld, sie ist aber auch im Mittelfeld möglich ${ }^{17}$ :

(38) a. VOTto ist ins Kino gegangen (und $\sqrt{\text { MarIA in die } 1 O p e r)}$

b. Mindestens $\sqrt{ }$ EIn Gemälde hat Maria heute UEdem Gast gezeigt

c. Heute hat Maria mindestens $\sqrt{ }$ EIn Gemälde VEdem Gast gezeigt

Die I-Topikalisierung ist mit bestimmten inhaltlichen Eigenschaften verbunden. Die beiden hervorgehobenen Positionen sind jeweils kontrastiert zu inhaltlichen Alternativen. Bei skopussensitiven Ausdrücken führt die Konstruktion zu einer Skopusbeziehung, die den strukturellen Verhältnissen der Grundpositionen der Ausdrücke entspricht. In (38b, c) resultiert dies in einer Skopusanordnung, die invers ist zu der Oberflächenreihenfolge der Ausdrücke.

In der Diskussion dieser Konstruktion wird hin und wieder suggeriert, daß es sich bei der ersten hervorgehobenen Konstituente notwendigerweise um ein Satztopik handelt (so Büring (1995)). Diese Sichtweise ist mit unseren Überlegungen nicht vereinbar. Zunächst zeigen $(38 \mathrm{~b}, \mathrm{c})$, daß ein Element, welches nicht referentiell ist, mit der für die sog. ITopikalisierung charakteristischen Betonung versehen werden kann. Ein solches Element kann nun aber auch unter dieser speziellen Betonung nicht in dem für Topiks reservierten Bereich des Satzes auftreten:

(39) a. Heute hat Maria offenbar mindestens $\sqrt{ }$ EIn Gemälde VEdem Gast gezeigt

b. ??Heute hat Maria mindestens $\sqrt{ }$ EIn Gemälde offenbar VEdem Gast gezeigt

16 So auch z.B. Jacobs (1984) und Molnár (1998)

17 Der Name I-Topikalisierung stammt von J, Jacobs. Jacobs setzt allerdings als Ziel dieser Bewegung (im Normalfall) das Vorfeld an (Jacobs (1996)). 
Dies zeigt, daß die I-topikalisierte Phrase nicht notwendigerweise Topikstatus hat. Natürlich kann die Wurzelkontour auch mit einem Topik-fähigen Element assoziiert werden. Ein solches Element kann dann auch im Topikbereich auftreten, d.h. es kann als Topik ausgezeichnet werden:

(40) Heute wird $\mathcal{V}$ Tto wahrscheinlich in die Oper gehen

Molnár (1998) kommt ebenfalls zu dem Ergebnis, daß die I-Topikalisierung unabhängig ist von der Auszeichnung eines Satztopiks. Sie spricht daher von I-Kontur.

(VIII) Oberhalb des Topikbereichs und unterhalb von $\mathrm{C}^{0}$ gibt es eine Position für Elemente mit I-Kontur und für kontrastfokusierte Elemente.

Im letzten Punkt wurde darauf hingewiesen, daß die Zuweisung der I-Kontur an eine Phrase nichts mit einer etwaigen Topikauszeichnung zu tun hat. Diese Behauptung wird durch ein weiteres Phänomen bestätigt.

Zwischen der Position des Komplementierers bzw. des finiten Verbs in V2-Sätzen und dem durch (6) charakterisierten Topikbereich befindet sich die Zielposition einer Bewegung, der Elemente mit I-Kontur unterzogen werden können ${ }^{18}$. In diese Position können auch kontrastfokusierte Eemente gestellt werden. Daß diese Zielposition für Elemente mit I-Kontur oder Kontrastfokus nicht zum Topikbereich gehört, erkennt man an einer Reihe von Fakten:

(41) a. Obwohl ver LIEren $_{1}$ Otto nie und NIMmer glaubte, jemals $t_{1}$ lernen zu müssen

b. da mindestens $\sqrt{ }$ EIn Gemälde heute erfreulicherweise UEder betrachtete

c. Weil GRÜN Hans die Tür gestrichen hat (und nicht ROT)

(41a) zeigt, daß diese Position Ziel einer Bewegung ist, die nicht Satz-gebunden ist (Brand et al. (1992)). In Abschnitt 8 wird in Punkt (viii) darauf hingewiesen, daß dies für die Topik-Voranstellung nicht zutrifft. Diese ist strikt Satz-gebunden. (41b) zeigt, daß in die fragliche Position Elemente mit I-Kontur plaziert werden können, die prinzipiell nicht Topik-fähig sind (vgl. (39b)). (41c) zeigt, daß unter Kontrastfokusierung ein Element in diese Position gestellt werden kann, das ohne spezielle Betonung ortsfest ist.

Es ist somit wichtig, den durch (6) benannten Topikbereich von der strukturell höher positionierten Zielposition der Bewegung unter I-Kontur oder Kontrastfokus strikt zu unterscheiden.

\section{Pronomen}

Wir wollen in diesem Abschnitt einen kurzen Blick auf Pronomen werfen. Dabei werden allerdings nur die für unser Thema unmittelbar relevanten Stellungen betrachtet. Es wird kein Versuch unternommen, die Syntax der Pronomen des Deutschen zu thematisieren.

Die kanonische Position eines unbetonten Pronomens im Mittelfeld ist vor einem Satzadverbial:

18 Haider \& Rosengren (1998) setzten ebenfalls eine solche Position nach der C-Position an. Sie nennen die Bewegung unter I-Kontur mit Zielposition im Mittelfeld 'T-Scrambling'. Diese Bezeichnung ist jedoch ebenso wie die Bezeichnung I-Topikalisierung nach den vorliegenden Überlegungen nicht ideal. Beide suggieren ja, daß es sich hierbei um die Voranstellung eines Topiks handelt. 
(42) weil er ihr wahrscheinlich das Buch gegeben hat

Aber es gibt auch Vorkommen von unbetonten Pronomen nach den Satzadverbialen (Lenerz (1993)) $)^{19}$ :

(43) a. weil wahrscheinlich gestern ein Mädchen es ihm gegeben hat

b. da Otto leider den Filmstar ihr nicht vorgestellt hat

Es sind nun einige der Tests aus Abschnitt 2 auf Pronomen anwendbar:

(44) Ich erzähl dir mal was von Otto .

a. Ich glaube, daß ihn 1 die Botschafterin erfreulicherweise heiraten wird

b. \#Ich glaube, daß erfreulicherweise die Botschafterin ihn, heiraten wird

(45) Ich habe etwas Neues über Hans 1 gehört.

a. Gestern hat erfreulicherweise Maria $i h n_{1}$ in der Stadt getroffen. Derselbige 1 hat sich sehr darüber gefreut

b. Gestern hat Maria ihn $_{1}$ erfreulicherweise in der Stadt getroffen. $*$ Derselbige $_{1}$ hat sich sehr darüber gefreut

Die Daten zeigen, daß die Pronomen, die vor einem Satzadverbial positioniert sind, als Satztopiks fungieren, während dies für Pronomen nach einem Satzadverbial nicht gilt ${ }^{20}$. Referentielle Pronomen verhalten sich demnach so, wie man es angesichts von (6) erwartet.

Betrachten wir Pronomen in der Präsentativkonstruktion:

(46) a. *Es hat er den Chef angerufen

b. Es hat ihn (erstaunlicherweise) der Chef angerufen

Wie in Abschnitt 3 unter Punkt (VI) erwähnt, ist die Präsentativkonstruktion nicht möglich mit einem topikalen Subjekt. Ein topikales Objekt ist in der Konstruktion jedoch möglich. Nach Lenerz (1993) tritt ein Subjektpronomen stets vor dem Satzadverbial auf. Hierfür steht zwar eine Erklärung noch aus, aber für uns ergibt sich daraus, daß ein Subjektpronomen, im Unterschied zu einem Objektpronomen, notwendigerweise topikalisch ist. Dies ergibt die Grammatikalitätsverteilung in (46).

Es kann nicht unerwähnt bleiben, daß auch nicht-referentielle Pronomen vor einem Satzadverbial positioniert werden:

(47) a. weil es wahrscheinlich schneien wird

b. weil sich gestern Hans bedauerlicherweise beschwert hat

Kann man für (47a) annehmen, daß sich das Expletiv vor dem Topikbereich adjazent zur C-Projektion befindet, so sind die Stellungsmöglichkeiten des Reflexivs eines inhärent reflexiven Verbs sehr vielfältig:

19 Nach Lenerz kann ein unbetontes Personalpronomen nach einem Satzadverbial nur adjazent zu einem Subjekt auftreten. Mir scheint allerdings auch (43b) akzeptabel

20 Damit widersprechen unsere Befunde der Behauptung von Erteschik-Shir (1997:21), wonach Pronomen notwendigerweise topikal seien. 
(48) a. weil Hans sich gestern bedauerlicherweise beschwert hat

b. weil Hans gestern bedauerlicherweise sich beschwert hat

c. weil offenbar keiner sich die Ausstellung anschauen will

d. weil die Ausstellung sich offenbar keiner anschauen will

Das Reflexivpronomen kann sich in vielen syntaktischen Nischen plazieren. Sein Vorkommen zwischen den beiden Topiks in (48a) oder zwischen einem Topik und dem Satzadverbial in (48d) sind daher weitere Belege seiner außerordentlichen syntaktischen Unrestringierheit, welche allerdings noch einer Erklärung harrt.

\section{Topiks und Generizität}

Mit Diesing (1992) rückten die Interpretationen indefiniter NPs ins Interesse der syntaktischen Diskussion: Abhängig von ihrer Position im Satz erhalten indefinite NPs unterschiedliche Deutungen. Nackte Plurale z.B. werden je nachdem, ob sie im Mittelfeld einem Temporaladverbial vorangehen oder folgen, unterschiedlich interpretiert:

(49) a. weil Väter an Weihnachten mit der Eisenbahn spielen

b. weil an Weihnachten Väter mit der Eisenbahn spielen

Die indefinite NP in (49a) kann nur generisch verstanden werden: Für Väter gilt typischerweise, daß sie an Weihnachten mit der Eisenbahn spielen. In (49b) hingegen hat diese NP auch eine existentielle Lesart: An Weihnachten gibt es Väter, die mit der Eisenbahn spielen. Daneben gibt es auch die generische Lesart für (49b).

Nach Diesing (1992) werden indefinite NPs außerhalb der VP 'stark' interpretiert (wobei die VP die Grundposition des Subjekts enthält). Die generische Interpretation ist eine der 'starken' Interpretationen, die existentielle Interpretation ist keine 'starke' Interpretation. ${ }^{21}$ Bei Diesing stellt somit die VP den Bereich von 'existential closure' dar, wobei bei ihr jede Art von Adverbial die VP-Grenze markiert. Nach Frey \& Pittner (1998) muß dieses Bild modifiziert werden. Es gibt durchaus bestimmte Adverbiale, die unterhalb der Grundposition des ranghöchsten Arguments basisgeneriert werden, z.B. Lokaladverbiale. Betrachten wir daher eine entsprechende Konstruktion mit einem Lokaladverbial:

\section{weil Väter im Park Fußball spielen}

Man stellt fest, daß für diesen Satz eine existentielle Lesart des Subjekts möglich ist, d.h. das Subjekt hat den Bereich der 'existential closure' noch nicht verlassen

Nach Frey \& Pittner (1998) wird aber z.B. ein Temporaladverbial oberhalb der Basisposition des ranghöchsten Arguments basisgeneriert. Eine Phrase, die einem Temporaladverbial vorangeht, steht daher nicht mehr im Bereich von 'existential closure'. Dies gilt etwa für das Subjekt von (49a). Es wird deshalb 'stark' interpretiert. Die Subjekte in (49b) und (50) hingegen befinden sich innerhalb der Domäne von 'existential closure'.

Eine andere Sichtweise der 'starken' Interpretation von indefiniten NPs vertreten Jäger (1996) und Erteschik-Shir (1997). Dieser Erklärungsansatz rekurriert auf das TopikKonzept. Nach Jäger und Erteschik-Shir ist eine 'starke' Interpretation von indefiniten

$21 \mathrm{Zu}$ den 'starken' Interpretationen von Indefinita sollen hier neben der generischen die spezifische und die partitive Interpretation gezählt werden. 
NPs nur dann möglich, wenn diese NPs Topiks sind. Auch die generische Interpretation eines nackten Plurals ist hiernach nur möglich, wenn dieser Topik ist. Zugrunde liegt diesem Ansatz die Setzung, daß die Deskription einer indefiniten NP nur dann in den Restriktor eines Operators geht, wenn diese NP topikalisch ist. Die generische Interpretation wird geknüpft an die Präsenz eines generischen Operators.

Wir müssen prüfen, ob sich dieser postulierte Zusammenhang zwischen Interpretation und Topikstatus mit unseren davon unabhängig entwickelten Topikkriterien bestätigen läßt. Es zeigt sich, daß dem nicht so ist: Eine indefinite NP kann eine generische Lesart haben, ohne Topik zu sein. Betrachten wir den folgenden Satz:

$$
\text { weil erfreulicherweise Väter an Weihnachten mit der Eisenbahn spielen }
$$

Das Subjekt weist hier die generische Lesart auf. Es ist höher als das Temporaladverbial positioniert und ist daher außerhalb des Bereichs von 'existential closure'. Zugleich steht es aber tiefer als das Satzadverbial, so daß wir mit (6) erwarten, daß es kein Topik ist. Nach (6) ist eine generisch interpretierte NP nur dann Topik, wenn sie vor dem Satzadverbial auftritt:

(52) weil Väter erfreulicherweise an Weihnachten mit der Eisenbahn spielen

Die folgenden Tests aus Abschnitt 3 bestätigen unsere Vorhersagen:

\section{'aboutness'-Kontext:}

(53) Da wir gerade von Vätern sprechen

a. \#Ich habe gehört, daß erfreulicherweise Väter an Weihnachten mit der Eisenbahn spielen

b. Ich habe gehört, daß Väter erfreulicherweise an Weihnachten mit der Eisenbahn spielen

\section{Das Bezugselement kataphorischer Pronomen:}

(54) a. *Weil sie ${ }_{1}$ ewig Kinder bleiben, spielen seltsamerweise Väter ${ }_{1}$ an Weihnachten mit der Eisenbahn

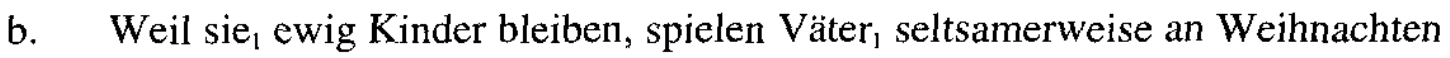
mit der Eisenbahn

\section{Demonstrativpronomen:}

(55) a. Die Regierung will alleinerziehende Väter erfreulicherweise im nächsten Jahr unterstützen. ??Dieselbigen haben es wirklich verdient.

b. Die Regierung will erfreulicherweise alleinerziehende Väter im nächsten Jahr unterstützen. Dieselbigen haben es wirklich verdient.

Die Tests zeigen, daß generisch interpretierte nackte Plurale, die vor einem Temporaladverbial, aber nach einem Satzadverbial auftreten, keine Topiks sind. Die generische Interpretation einer indefiniten NP ist somit keine hinreichende Bedingung für ihren Status als Topik.

Der gleiche Befund ergibt sich für andere sogenannte 'starke' Interpretationen indefiniter NPs. Hierzu wird die partitive Interpretation einer indefiniten NP mit einem Numeral gezählt: 
(56) a. Hans hat drei Einhörner heute gefüttert

b. Hans hat heute drei Einhörner gefüttert

In (56b) weist das Objekt eine rein existentielle Lesart auf. Dies ist nicht möglich in (56a). Dieser Satz kann aber so verstanden werden, daß von drei Einhörnern aus einer gegebenen Menge die Rede ist.

Jäger (1999) will auch diese Lesart mit dem Topikstatus der indefiniten NP begründen. Wiederum ist dieser Erklärungsansatz mit unseren Überlegungen nicht kompatibel:

(57) Hans hat erfreulicherweise drei Einhörner heute gefüttert

Das Objekt in (57) hat die partitive Lesart, jedoch ist es nicht Topik:

(58) a. *Ihre ${ }_{1}$ Pfleger haben glücklicherweise drei Einhörner ${ }_{1}$ heute gefüttert

b. Ihre ${ }_{1}$ Pfleger haben drei Einhörner ${ }_{1}$ glücklicherweise heute gefüttert

(59) [A:] Erzähl doch mal was von den Einhörnern.

[B:] a. \#Ich denke, daß Otto glücklicherweise drei Einhörner heute gefüttert hat

b. Ich denke, daß Otto drei Einhörner glücklicherweise heute gefüttert hat

Wir schließen aus diesen Beobachtungen, daß auch aus dieser 'starken' Interpretation einer indefiniten NP nichts über ihrer etwaigen Topikstatus folgt. ${ }^{22}$

Dieser Befund wird durch ein weiteres Datum bestätigt. Viel diskutiert wurde in den vergangenen Jahren auch der Umstand, daß ein nackter Plural als Subjekt gewisser Prädikate nur generisch und nicht existentiell interpretiert werden kann (s. z.B. Diesing $(1992))^{23}$

(60) weil Frauen zielstrebig sind

Diesen Tatbestand wollen Jäger (1996), Erteschik-Shir (1997) durch die axiomatische Festsetzung ableiten, daß jeder Satz mindestens ein Satztopik aufweist. Die Überlegung ist die folgende: Das Satztopik kann im Prinzip lexikalisch unrealisiert sein. Dies ist nach Ansicht der Autoren der Fall, wenn das von einem Satz bezeichnete Ereignis als Satztopik fungiert. Die Autoren setzen zudem fest, daß ein Zustand nicht Topik sein könne. Die Prädikate, die die Interpretation wie in (60) zeigen, bezeichnen einen Zustand. Wenn sie kein weiteres Argument haben, muß daher nach Voraussetzung ihr Subjekt notwendigerweise Topik sein. Ist dies ein nackter Plural, muß dieser somit generisch interpretiert werden.

Dies ist eine Argumentationskette, die mit unseren Überlegungen nicht verträglich ist. Zwar wird ein nackter Plural als Subjekt dieser Prädikate notwendigerweise generisch interpretiert, aber ein solches Subjekt ist deshalb nicht notwendigerweise topikal (vgl.

22 Da eine NP im Topikbereich den Bereich von 'existential closure' verlassen hat, gilt natürlich die andere 'Richtung': Wenn eine indefinite NP Topik ist, dann kann sie nur 'stark' interpretiert werden (s. z.B. (52)). Eine 'stark' interpretierte indefinite NP erfüllt die Bedingung an Topiks, eindeutig verankerbar zu sein. Auch generisch interpretierte Ausdrücke als referentiell und daher als Topik-fähig in unserem Sinn aufzufassen ist seit Carlson (1978) eine gängige Vorstellung.

23. Ursprünglich wurde angenommen, daß dies genau für die Prädikate gilt, die permante, essentielle Eigenschaften ausdrücken. Diese wurden von Carlson (1978) 'individual-level'-Prädikate (IL-Prädikats) genannt. Inzwischen ist jedoch bekannt, daß die Korrelation in dieser einfachen Weise nicht zutrifft (s. z.B. Jäger (1999a)). 
(61a)). Auch eine definite NP als Subjekt eines solchen Prädikats muß nicht im Topikbereich auftreten (vgl. (61b)).

(61) a. weil glücklicherweise Frauen zielstrebig sind

b. weil gliicklicherweise Maria sehr intelligent ist

Durch diese Beobachtung ergibt sich auch, daß die Interpretation von (60) keine Evidenz ergibt für das Postulat, jeder Satz besitze ein (explizites oder implizites) Satztopik. Wenn man trotzdem die Notwendigkeit eines Satztopiks annehmen möchte ${ }^{24}$, dann muß man aufgrund von (61) die Möglichkeit eines impliziten Topiks sowohl für Sätze vorsehen, die ein Ereignis beschreiben, als auch für solche, die einen Zustand beschreiben. Dies erschiene mir auch natürlicher als nur für Ereignisse einen potentiellen Topikstatus anzusetzen.

Seit Diesing wird häufig angenommen, daß die Subjekte jener Prädikate, die eine 'starke' Lesart ihres Subjekts erzwingen, in der Spec-Position von IP basigeneriert werden, während Subjekte anderer Prädikate VP-intern basisgeneriert sind. Wenn man dies annehmen möchte, folgt im Rahmen unsere Befunde, daß die Spec-Position von IP 'unterhalb' der Position der Satzadverbiale, d.h. unterhalb des Topikbereichs, anzusetzen ist. Dies bedeutet somit, daß die Annahme in Drubig (1992:174)), ein VP-externes Subjekt sei generell mit der 'aboutness'-Lesart assoziiert, nicht aufrecht erhalten werden kann.

Zusammenfassend läßt sich feststellen, daß das Vorgehen einiger Autoren, die 'starke' Interpretation einer indefiniten NP als hinreichende Bedingung für ihren Topikstatus anzusetzen, mit unseren Topikkriterien nicht verträglich ist.

\section{Welche Elemente kommen als Satztopik in Frage?}

Bislang haben wir bei unseren Überlegungen in erster Linie NPs in Argumentfunktion betrachtet. Aber auch andere Elemente kommen im Deutschen als Satztopik in Frage.

Betrachten wir PPs als Argumente und als Adjunkte:

(62) a. weil Hans mit Maria sicherlich gut bekannt ist

b. da heute in der Küche leider jemand gestürzt ist

Die Beispiele zeigen, daß nach (6) sowohl Argument-PPs als auch Adjunkt-PPs als Satztopiks möglich sind. ${ }^{25}$

Adverbien können als Satztopiks auftreten. Natürlich ist dies nur möglich, wenn sie referentiell sind. Man vergleiche (63a) und (b):

24 Z.B. um festsetzen zu können, daß jede Aussage die Prädikation eines Kommentars über einen Satzgegenstand darstellt.

25 Die Präposition eines Arguments wie in (62a) kann als semantisch leer aufgefaßt werden. Jedoch wirft der topikale Status einer PP wie in (62b) ein Problem auf, auf das C. Maienborn hingewiesen hat. Die PP in diesem Satz denotiert die Eigenschaft, im Innern der Küche lokalisiert zu sein. Aber der Aussagegegenstand von (62b) ist nicht diese Eigenschaft, sondern die Innenregion der Küche. Aus diesem Grund nimmt Jacobs (1999) an, daß die semantische Repräsentation einer solchen PP ein referentielles Argument enthält, das diese Innenregion denotiert. Als Evidenz für diese Argumentstelle verweist Jacobs auf Phrasen wie 'überall in der Küche', bei denen auf die fragliche Argumentstelle zugegriffen wird. 
(63) a. Otto muß morgen hier wahrscheinlich eine Rede halten

b. *Otto muß oft wahrscheinlich eine Rede halten

Auch Sätze sind mögliche Satztopiks:

(64) Hans hat im letzten Jahr [dieses Buch zu lesen] anscheinend mehrmals versucht

Finite Sätze sind im Mittelfeld schlecht umzustellen. Für sie kann daher (6) nicht direkt zur Anwendung gelangen. Aber Test (III) aus Abschnitt 3 läßt vermuten, daß finite Sätze potentielle Satztopiks sind ${ }^{26}$ :

(65) Alle haben es ${ }_{1}$ behauptet. [Daß Maria unschuldig ist $]_{1}$ hat daher auch Otto geglaubt.

Wie verhalten sich Elemente in prädikativer Funktion? Sie lassen sich (marginal) vor ein Satzadverbial stellen, s. (66a, b). Die Voranstellung vor ein Satzadverbial ist besser als eine sonstige beliebige Umstellung im Mittelfeld, s. (66c):

(66) a. (?)da Hans Politiker erstaunlicherweise geworden ist

b. (?)da Hans bescheiden erfreulicherweise geblieben ist

c. *da Hans erfreulicherweise bescheiden trotz des Ruhms geblieben ist

Eine Konstruktion, die als Paradebeispiel für eine Konstruktion gilt, die ein 'aboutness'Topik auszeichnet, ist die Linksversetzung (z.B. Jacobs (1999)). Ein prädikatives Element ist in dieser Konstruktion möglich:

(67) Bescheiden, das ist Hans erfreulicherweise immer geblieben

Die Beispiele (66a, b) und (67) legen somit nahe, daß auch prädikative Elemente Topikfähig sind. Dies weist darauf hin, daß Eigenschaften als Objekte konzeptualisiert werden können ${ }^{27}$.

\section{Ein Blick auf einige andere Vorschläge}

In diesem Abschnitt soll vergleichend auf einige neuere Arbeiten eingegangen werden, die für unser Thema unmittelbar einschlägig sind.

Vallduví (1992), Vallduví \& Engdahl (1996) schlagen eine dreiteilige hierarchische Informationsgliederung der Sätze vor:

26 Sätze können auch in der sogenannten Null-Topik-Konstruktion wegfallen, von der angenommen wird, daß sie nur Topiks betreffen kann:

(i) [A:] Der VfB Stuttgart hat wieder verloren

[B:] $\varnothing$ ist doch jedem schon bekannt

27 Man beachte, daß ein Adjektiv in der Funktion eines Adverbials der Art \& Weise in der Linksversetzung nicht möglich ist (Jacobs (1999)):

(i) *Sorgfältig so hat Otto das Buch gelesen

Ein solches Adverbial ist nicht Topik-fähig. Dies läßt vermuten, daß ein Adverbial der Art \& Weise von einem höheren semantischen Typ ist als Prädikative. 
Vallduvís 'link'-Konzept nimmt Reinharts Charakterisierung der Topiks auf. Der 'link' eines Satzes gibt nach Vallduví dem Hörer an, wo er in seinem Informationszustand die Information des Satzes abspeichern soll. Vallduví setzt einen 'link' als Teil des Hintergrunds an. Mit 'tail' wird der Rest des Hintergrunds bezeichnet. Nach Vallduví ist ein 'link' stets Satz-initial. Desweiteren folgert er aus seiner Charakterisierung eines 'links', daß ein solcher nur dann ausgezeichnet wird, wenn ein im Vergleich zum Vorsatz neues Element als Gegenstand der Satzaussage gesetzt wird.

Vallduvís 'link'-Konzept ist mit unseren Beobachtungen zum Satztopiks im Deutschen nicht vereinbar. Zunächst ist festzustellen, daß das Satztopik im Deutschen auch dann strukturell ausgezeichnet wird, wenn der Gegenstand der Satzaussage bezüglich des Vortextes gleich bleibt.

(69) In unserer Firma ist Hans 1 wahrscheinlich der beliebteste Kollege.

a. Jedoch wird der nette $\operatorname{Kerl}_{1}$ leider die Firma bald verlassen.

b. \#Jedoch wird leider der nette $\mathrm{Kerl}_{1}$ die Firma bald verlassen.

Ebenfalls im Widerstrcit steht Vallduvís Annahme, daß der 'link' stets Teil des Hintergrundes ist. In Abschnitt 3 wurcle unter (VI) darauf hingewiesen, daß ein Satztopik im Fokusbereich eines Satzes auftreten kann. Deshalb ist es nicht möglich, die beiden Zweiteilungen Fokus-Hintergrund und Topik-Kommentar auf eine Dreiteilung zu reduzieren, wie dies Vallduví versucht. Schließlich ist Vallduvís Festlegung, 'links' seien stets Satz-initial, offensichtlich auf die durch (6) charakterisierten Satztopiks nicht übertragbar.

Meinunger (2000) betrachtet in seiner Untersuchung die Positionierung der Argumente im deutschen Mittelfeld. Er deutet jede Form von Scrambling im Deutschen als Voranstellung von Phrasen, die Topikstatus aufweisen. Desweiteren nimmt er an, daß topikale Phrasen gescrambelt werden müssen. Dabei ist Meinunger dem 'familiarity'Konzept von Topik verpflichtet.

Das erste Problem aus unserer Sicht ist, daß Meinunger annimmt, daß sämtliche Adverbiale oberhalb der Grundpositionen der Argumente positioniert sind. Daraus folgt, daß alle Elemente, die im Mittelfeld irgendeinem Adverbial vorangehen, Topiks sein sollten. Es wurde in Abschnitt 3 unter (I) gezeigt, daß diese Analyse mit unserer Konzeption von Topik unvereinbar ist.

Problematisch für Meinungers Ansatz sind desweiteren Beispiele wie die folgenden:

(70) a. Otto wird wohl irgendetwas irgendjemandem zeigen wollen

b. In dieser Woche hat Otto mindestens eine Briefmarke jeder Besucherin gezeigt

In (70) stehen die Akkusativ-NPs nicht in ihrer Grundposition. Ihre Umstellung kann aber schwerlich mit Topikalität in Verbindung gebracht werden. Es erscheint daher nicht plausibel, Scrambling generell als Voranstellung von Topiks zu deuten.

Schließlich sind Daten wie in (30) des Abschnitts 3 im Rahmen von Meinungers Annahmen nicht erwartet. Anaphorische Phrasen sind 'familiar'. Als solche sollten sie nach Meinunger topikal sein, und sie sollten bewegt werden. Daß die anaphorischen Phrasen in (30) topikal seien, wurde bereits in Abschnitt 3 bestritten. Der folgende Beispiel zeigen sogar, daß anaphorische Phrasen in ihrer Grundposition verharren können: 
(71) a. Vor einer Woche wurde ein Mann unter Tatverdacht verhaftet. Laut Polizei hat heute wer dem Verdächtigen zur Flucht verholfen.

b. Hans hat eine Menge Photos von Italien. Heute abend will er nun wieder netten Damen seine Fotos zeigen

W-Indefinita sind ortsfest ${ }^{28}$, daher muß auch die nachfolgende Dativ-NP des zweiten Satzes von (71a) in der Grundposition stehen. Die existentielle Interpretation des indirekten Objekts im zweiten Satz von (71b) zeigt, daß die Phrase die Domäne von 'existential closure' nicht verlassen hat. Es ist anzunehmen, daß sie in ihrer Grundposition steht. Damit steht aber auch die nachfolgende anaphorische Phrase in ihrer Grundposition.

Jacobs (1999) gibt vier Eigenschaften an, die er mit prototypischen TopikKommentar-Strukturen assoziiert sieht:

(72) (i) Das Topik ist informationsstrukturell separiert.

(ii) Das Topik spezifiziert eine Argumentstelle eines Prädikats im Kommentar, und es wird von keinem anderen Element mit dieser Eigenschaft auf der SStruktur k-kommandiert.

(iii) Das Topik gibt die Adresse an, unter der die Information des Satzes abgelegt wird.

(iv) Das Topik gibt den Bereich an, relativ zu dem der Gültigkeitsanspruch der Proposition eingeschränkt wird.

Bedingung (i) verweist auf die Tatsache, daß ein Satz mit Topik-Kommentar-Gliederung in zwei Schritten prozessiert wird (s. Abschnitt 1), wobei Jacobs annimmt, daß in ikonischer Entsprechung sowohl das Topik als auch der Kommentar einen Hauptakzent aufweisen. Nach der Bedingung (ii) steht ein prototypisches Topik an der Spitze des Satzes, d.h. außerhalb des Mittelfeldes. Bedingung (iii) entspricht der Charakterisierung der 'aboutness'-Topiks durch Reinhart (1981). Die Eigenschaft (iv) betrifft sogenannte Rahmen-setzende Ausdrücke wie in folgenden Beispielen:

(73) a. weil im Mittelalter Mönche viel Bier tranken

b. Im Fall eines Sieges wird die Mannschaft eine Belobigung erhalten

c. Gesundheitlich geht es Peter gut

Diese Phrasen werden in der Literatur unter dem Stichwort 'Frame Topics' diskutiert ${ }^{29}$. Wie die Charakterisierung in (iv) zeigt, sind sie zu unterscheiden von 'aboutness'Topiks.

Entscheidend für Jacobs Überlegungen ist die Annahme, daß in der Regel in einer bestimmten syntaktischen Konstruktion, die in der Literatur mit der Topik-KommentarGliederung in Verbindung gebracht wird, das Topik nicht sämtliche, sondern nur einige Eigenschaften von (72) erfüllt. Die unterschiedlichen Topik- Konstruktionen sind daher lediglich durch Familienähnlichkeit aufeinander bezogen. ${ }^{30}$

Unsere Beobachtungen zur Auszeichnung der Topiks im Mittelfeld fügen sich nicht unmittelbar ein in die Charakterisierungen von Jacobs. Denn lediglich die Eigenschaft (iii) von (72) trifft für die Topik-Auszeichnung im Mittelfeld nach (6) zu.

28 Vgl. z.B. Haider \& Rosengren (1998).

29 Siehe für das Deutsche insbesondere Maienborn (1998).

30 Jacobs illustriert seine Überlegungen an Hand dreier Konstruktionen: Linksversetzung, ITopikalisierung und freies Thema. 
Beginnen wir mit Eigenschaft (iv). Ein im Topikbereich nach (6) auftretendes Element muß natürlich kein rahmensetzender Ausdruck sein. (iv) ist aber auch keine hinreichende Bedingung für die Positionierung nach (6). Ein rahmensetzender Ausdruck tritt nur fakultativ im Topikbereich auf:

(74) a. weil im Mittelalter erstaunlicherweise Mönche viel Bier tranken b. weil erstaunlicherweise im Mittelalter Mönche viel Bier tranken

Und auch dies ist nur möglich für einen referentiellen rahmensetzenden Ausdruck:

(75) a. *weil zu keiner Zeit erfreulicherweise Mönche viel Bier tranken b. weil erfreulicherweise zu keiner Zeit Mönche viel Bier tranken

Dies zeigt nochmals, daß (6) 'aboutness'-Topiks charakterisiert. Ein Rahmen-setzender Ausdruck kann zwar zum Gegenstand der Satzaussage werden, aber nur, wenn er die Forderung an 'aboutness'-Topiks nach Verankerbarkeit erfüllt.

Die Eigenschaft (i) verbindet Jacobs mit einer intonatorischen Separierung. Die intonatorischen Separierung kann bei 'Mittelfeldtopiks' erfüllt sein, aber sie muß es nicht. $\mathrm{Da}$ die Eigenschaft (ii) für die 'Mittelfeldtopiks' nicht erfüllt ist, ist unmittelbar klar.

In Haftka (1995) und Zimmermann (1999) wird angenommen, daß Satzadverbiale im Mittelfeld des deutschen Satzes die Grenze ziehen zwischen gegebenen Einheiten (Hintergrund) und neue Information beinhaltenden Einheiten (Fokus). Satzadverbiale kkommandieren hiernach im Normalfall minimal den fokalen Bereich des Satzes. Dieser wird mit der VP identifiziert. Gegen diese Annahme spricht zunächst, daß in einem 'all focus'-Satz ein Satzadverbial auftreten kann:

(76) Was ist passiert?

a. Heute hat Hans Maria erstaunlicherweise den Ferrari überlassen

b. Heute hat ein Fan aus Stuttgart leider einen Fan aus Berlin verprügelt

Ein Satzadverbial muß aber auch nicht an der Grenze einer nur einen Teil des Satzes umfassenden Fokusdomäne stehen, wie (36) zeigt, hier wiederholt:

(36) a. Was wird Maria heute wahrscheinlich mit Otto machen?

b. [Hintergr. Heute wird Maria wahrscheinlich Otto] [Fokus durch Kreuzberg führen]

Ein Problem mit Haftkas Ansatz ist weiterhin, daß sie anaphorische NPs stets aus der VP herausbewegen möchte, da diese 'bewußtseinspräsent' seien. Die oben in (71) angeführten Beispiele stellen daher auch für ihre Annahmen ein Problem dar.

\section{Syntaktische Eigenschaften der Topik-Voranstellung im Vergleich zu Scrambling und Vorfeldbesetzung}

Die Positionierung eines Topiks vor ein Satzadverbial im Mittelfeld ist nach Frey \& Pittner (1998) Resultat einer Bewegung. Mit dem Terminus Scrambling bezeichnet man gemeinhin beliebige Umstellungen von Satzgliedern im Mittelfeld. Es stellt sich somit die Frage, ob die Topik-Voranstellung eine Instanz von Scrambling ist, d.h., ob die Eigen- 
schaften der Topik-Voranstellung die gleichen sind wie die Eigenschaften sonstiger Umstellungen im Mittelfeld. Daher soll nun die Topik-Voranstellung verglichen werden mit Umstellung von Elementen rechts vom Satzadverbial (nur letzteres soll im folgenden unter 'Scrambling' verstanden werden). Parallel dazu soll geprüft werden, ob die TopikVoranstellung einer Phrase Eigenschaften mit der Bewegung einer solchen Phrase ins Vorfeld teilt. ${ }^{31}$

(i) Topik-Voranstellung induziert wie Scrambling und Vorfeldbesetzung kein weakcrossover.

Die Voranstellung einer Phrase in den Topikbereich über ein koindiziertes Pronomen hinweg führt zu keiner Grammatikalitätsverletzung, d.h. sie induziert keinen weakcrossover -Effekt, (77a). Das gleiche gilt aber auch für Scrambling, (77b), und für Vorfeldbesetzung, $(77 \mathrm{c})$ :

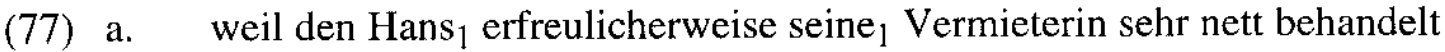

b. weil erfreulicherweise den Hans 1 seine, Vermieterin sehr nett behandelt

c. den Hans ${ }_{1}$ behandelt seine ${ }_{1}$ Vermieterin sehr nett

Aus dem fehlenden weak-crossover können also keine weiterreichenden Schlüsse über den Status der Topik-Voranstellung gezogen werden, zumal im Deutschen die Satzinterne Umstellung selbst einer quantifizierten Phrase zu keiner weak-crossoverVerletzung führt:

(78) a. weil in dieser Stadt jeden Studenten $_{1}$ seine $_{1}$ Vermieterin freundlich behandelt

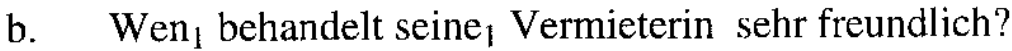

(ii) Topik-Voranstellung wird durch einen bestimmten Trigger ausgelöst. Ein entsprechender einfacher Trigger für Scrambling ist nicht bekannt.

Nach (6) müssen die topikalen Phrase des Mittelfeldes und nur diese der TopikVoranstellung unterzogen werden. Eine entsprechende eindeutige Auszeichnung der Phrasen, die Scrambling unterzogen werden, ist bislang nicht bekannt. ${ }^{32}$ Es wird zwar hin und wieder vorgeschlagen (z.B. von Diesing (1997)), daß die 'starke' Interpretation einer indefiniten NP bzw. die Tatsache, daß eine NP Bekanntes denotiert, der Trigger für Scrambling sei. Die Beispiele in (49b) und in (70) widerlegen jedoch diese Annahme. Es ist natürlich möglich, daß in der Zukunft eine Eigenschaft entdeckt wird, die alle gescrambelte Phrasen teilen. Aber diese wird, wenn (6) richtig ist, verschieden sein von der Topikeigenschaft.

31 Ein Vergleich mit der unter (VIII) in Abschnitt 3 angesprochenen 'Mittelfeldbewegung' unter I-Kontur bzw. Kontrastfokus in eine Position oberhalb des Topikbereichs wird im folgenden nicht durchgeführt. Zum einen sind deren Eigenschaften noch zu wenig bekannt, zum anderen hat diese Bewegung informationstheoretische Effekte, die von ganz anderer Art sind als die von uns thematisierten.

32 Allerdings gehen Haider \& Rosengren (1998:9) zu weit, wenn sie schreiben: "... the interpretation effect that is induced by Scrambling is found in unscrambled structures as well." Es gibt z.B. Skopusoptionen, die erst aufgrund von Scrambling entstehen:

(i) da er mindestens ein Gedicht, fast jeder Dame $t_{1}$ vortrug

Der Satz besitzt eine Lesart mit weitem Skopus der existentiell quantifizierten Phrase. Stünde die Phrase in ihrer Grundposition, wäre diese Lesart nicht gegeben. Daneben besitzt der Satz auch eine Lesart mit weitem Skopus des nicht-bewegten allquantifizierten Objekts. 
Wenn im Mittelfeld kein Satzadverbial auftritt, ist u.U. an der Oberfläche nicht erkennbar, ob eine Topik-fähige Phrase im Topikbereich steht oder nicht. In einem solchen Fall liefern unsere Tests Evidenz für beide Analysen:

(79) a. Da er 1 eine vorzügliche Dissertation geschrieben hat, wird Fred ${ }_{1}$ einen Preis bekommen

b. Es wird heute Fred einen Preis bekommen.

In (79a) wird das Subjekt als Topik analysiert (vg. (III) in Abschnitt 2), in (79b) wird es als Nicht-Topik analysiert (vgl. (VI) in Abschnitt 2). Natürlich kann auch bei Fehlen eines Satzadverbials die Struktur informationstrukturelle Eindeutigkeit aufweisen. Eine Phrase kann z.B. nicht Topik sein, wenn ihr eine nicht Topik-fähige Phrase vorangeht, denn dann kann sie selbst nicht im Topikbereich stehen ${ }^{33}$ :

(80) *Sein 1 Vater kann an keinem Tag den Peter 1 von der Schule abholen

(iii) Es gibt Elemente, die der Topik-Voranstellung unterzogen werden können, die aber sonst keine Mittelfeldumstellung erlauben.

Im Deutschen gibt es Elemente, die sich der Umstellung im Mittelfeld zu widersetzen scheinen. Dazu gehören verbnahe Dative und Genitive:

(81) a. *weil Hans bedauerlicherweise dieses Anschlages einen Unschuldigen bezichtigte

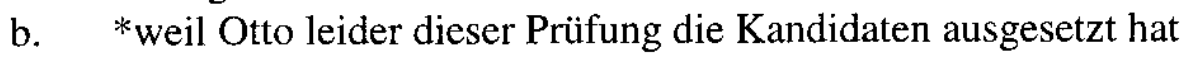

Interessanterweise lassen sich diese Elemente aber in den Topikbereich stellen:

(82) a. weil Hans dieses Anschlages bedauerlicherweise einen Unschuldigen bezichtigte

b. weil Otto dieser Prüfung leider die Kandidaten ausgesetzt hat

Die fraglichen Elemente können auch der Vorfeldbesetzung unterzogen werden.

In Abschnitt 6 wurde darauf hingewiesen, daß prädikative Elemente tendenziell den gleichen Unterschied zwischen der Möglichkeit von Topik-Voranstellung und der Unmöglichkeit von Scrambling zeigen (vgl. (66)).

Kontraste wie diese sind schwer zu erfassen, wenn man wie Hetland (1992), Laenzlinger (1998) oder Haider \& Rosengren (1998) annimmt, daß Satzadverbiale im Mittelfeld überall basisgeneriert werden könnten. Denn es ist nicht klar, warum eine Umstellung dadurch grammatisch werden sollte, daß eine von der Umstellung unabhängige Konstituente zwischen die fraglichen Konstituenten plaziert wird. Nach Frey \& Pittner (1998) hingegen haben die Satzadverbiale im Mittelfeld eine bestimmte Grundposition, und diese ist sehr hoch. Wenn die Satzadverbiale eine Grundposition besitzen, eröffnet dies die Möglichkeit, Bewegung in eine Position vor den Satzadverbialen prinzipiell zu unterscheiden von sonstiger Mittelfeldumstellung.

33 Im folgenden Satz soll das Temporaladverbial mit flacher Betonung gesprochen werden. Unter Wurzelbetonung könnte es in der in Abschnitt 3 unter (VIII) identifizierten Position vor dem Topikbereich stehen. In diesem Fall könnte das Objekt im Topikbereich stehen. 
(iv) In einer Verb-Projektion im Vorfeld ist kein Topik möglich, Scrambling ist hier möglich.

Die Frage, ob Topiks im komplexen Vorfeld auftreten können, d.h. ob sie dort gemeinsam mit einem verbalen Element stehen können, kann nicht direkt mit Hilfe von (6) beantwortet werden. Der Grund ist, daß Satzadverbiale generell im komplexen Vorfeld nicht möglich sind (Frey \& Pittner (1998)):

(83) a. ihre Theorie erklären wird sie uns hoffentlich heute

b. *hoffentlich ihre Theorie erklären wird sie uns heute

c. *hoffentlich erklären wird sie uns heute ihre Theorie

Kriterien aus Abschnitt 2 sind jedoch anwendbar:

(84) a. Ich erzähl dir was von Otto. \#den Otto/ihn treffen werde ich an Ostern

b. $\quad *$ [den Otto jedenfalls]/[ihn jedenfalls] treffen werde ich an Ostern

c. $\quad *\left[\right.$ Volvos $_{1}$ nur blaue $\mathrm{t}_{1}$ kaufen $]$ wird auch Peter

Weder eine topikale lexikalische Phrase noch ein topikales Pronomen können im komplexen Vorfeld plaziert werden. Topik-Voranstellung innerhalb des komplexen Vorfelds ist somit nicht möglich. Scrambling hingegen ist hier möglich, wie (85b) zeigt:

(85) a. Kindern den Sternenhimmel erläutert hat er schon oft

b. den Sternenhimmel ${ }_{1}$ Kindern $_{t_{1}}$ erläutert hat er schon oft

(v) Topik-Voranstellung kann wie Scrambling mehrmals angewandt werden. Dies ist nicht möglich bei Vorfeldbesetzung.

Dies wurde bereits in Abschnitt 3 unter (III) hervorgehoben. Man beachte, daß die Topiks in unterschiedlicher Reihenfolgen auftreten können:

(86) a. weil heute Peter der Maria erfreulicherweise das Buch ausleihen wird

b. weil Peter der Maria heute erfreulicherweise das Buch ausleihen wird

Nach É. Kiss (1995a) gilt für die meisten Sprachen, die Topiks positionell auszeichnen, daß sie mehrere Topiks pro Satz zulassen und daß deren Ordnung frei ist.

Die Iterierbarkeit in unterschiedlicher Reihenfolge gilt auch für Scrambling, aber sie gilt nicht für die Vorfeldbesetzung. Das Vorfeld kann nur durch eine Phrase besetzt werden.

(vi) Topiks im Mittelfeld sind wie gescrambelte Phrasen transparent für Extraktion. Dies gilt nicht für Phrasen im Vorfeld.

Ist eine Phrase für Extraktion durchlässig, so bleibt diese Eigenschaft erhalten, wenn sich die Phrase im Topikbereich des deutschen Mittelfeldes befindet:

(87) a. Über Linguistik ${ }_{1}$ hätte Otto [einen solch schönen Artikel $t_{1}$ ] leider niemals verfaßt

b. Was ${ }_{1}$ hat Hans heute [ $\mathrm{t}_{1} \mathrm{zu}$ lösen] leider vergeblich versucht 
Aus einem Verbzweit-Satz kann nichts herausbewegt werden. Dies gilt auch für eine Subkonstituente der Phrase im Vorfeld ${ }^{34}$ :

(88) *Über Linguistik ${ }_{1}$ meint Karl [[einen solch schönen Artikel $\left.t_{1}\right]_{2}$ hätte Otto $t_{2}$ niemals verfaßt]

(vii) Topik-Voranstellung induziert wie Scrambling und im Unterschied zu Vorfeldbesetzung keine Barriere.

Die Topik-Voranstellung einer Phrase im Mittelfeld blockiert weder eine darüber hinweggehende Topik-Voranstellung einer anderen Phrase noch irgendeine andere Bewegung. Hingegen ist, wie bereits im letzten Punkt vermerkt, keine Bewegung aus einem Verbzweit-Satz möglich:

(89) a. Maria wird das Buch dem Hans $_{1}$ erfreulicherweise $t_{1} t_{2}$ ausleihen

b. *Das Buch 2 hoffe ich [dem Hans ${ }_{1}$ wird Maria $t_{1} t_{2}$ ausleihen]

(viii) Topik-Voranstellung ist wie Scrambling und ungleich Vorfeldbesetzung Satzgebunden.

Es ist nicht möglich, eine Phrase aus einem eingebetteten Satz in den Topikbereich des übergeordneten Satzes zu bewegen:

(90) *daß Eva dem Hans ${ }_{1}$ wahrscheinlich glaubt, [ $\mathrm{t}_{1}$ helfen zu müssen]

Dies steht im direkten Gegensatz zur Vorfeldbesetzung, welche nicht Satz-gebunden ist:

(91) a. Ich erzähl dir mal was von Otto.

Den Otto 1 behauptet Maria, [daß eine Norwegerin $\mathrm{t}_{1}$ heiraten wird]

b. Dieses Buch, das 1 hat Maria mich überredet [ $t_{1}$ zu kaufen]

In (91a) wird der Test (I) aus Abschnitt 2 durch ein Element, welches lang bewegt wurde, erfüllt. In (91b) ist das resumptive Pronomen der Linksversetzungskonstruktion lang bewegt worden. Es wurde bereits oben darauf hingewiesen, daß die Linksversetzung als Paradebeispiel einer Topikkonstruktion gilt (vg. Jacobs (1999)).

(ix) Topik-Voranstellung und Vorfeldbesetzung unterscheiden sich in den kontextuellen Anforderungen

Die bislang betrachteten Eigenschaften sind hauptsächlich syntaktischer Natur. Daneben gibt es aber einen primär pragmatischen Unterschied, dem wir uns jetzt zuwenden wollen. Betrachten wir die folgenden Beispiele:

(92) a. Vor zwei Tagen hat einigen Rentnern angeblich die Polizei Handschellen angelegt

b. Einigen Rentnern behauptet Maria, daß die Polizei Handschellen angelegt hat

34 Diese Beobachtung ist für unsere Gegenüberstellung nur relevant, wenn man der Standardannahme folgt, daß es im Deutschen Verbzweit-Sätze mit Komplementstatus gibt und daß Extraktion aus ihnen syntaktisch blockiert wird. Gewichtige Argumente gegen diese Annahmen finden sich in Reis (1997). Derselbe Vorbehalt gilt gegenüber der Beobachtung bezüglich Vorfeldbesetzung im folgenden Punkt (vii). 
c. Heute hat im Eisbärbecken erstaunlicherweise ein Zoobesucher ein Bad genommen

d. Im Eisbärbecken behauptet Karl, daß ein Zoobesucher ein Bad genommen hat

Die Sätze (92a) und (c) sind zwar markiert, da die Satzglieder im Mittelfeld in nichtkanonischer Reihenfolge auftreten. Aber die Sätze verlangen nicht notwendigerweise einen speziellen textlichen oder situativen Bezug. Dies ist anders für (92b) und (d). Damit diese Sätze angemessen sind, müssen die Phrasen im Vorfeld von (92b, d) auf Gegebenes bezogen werden können ${ }^{35}$. Passende Kontexte könnten z.B. sein:

(93) a. Die älteren Herrschaften wurden ganz wild. Einigen Rentnern behauptet Maria, daß die Polizei Handschellen angelegt hat

b. Im Berliner Zoo passiert doch immer wieder verblüffendes. Im Eisbärbecken behauptet Otto, daß ein Zoobesucher ein Bad genommen hat

In $(93 a, b)$ wird durch die Vorfeldphrase des zweiten Satzes jeweils ein Objekt bezeichnet, das Element einer Menge ist, welche im ersten Satz eingeführt wird ${ }^{36}$. Die ElementMenge-Beziehung ist eine der Möglichkeiten der kontextuellen Bezugnahme, die Identitätsbeziehung eine weitere. Es soll hier nicht der Versuch gemacht werden, zu bestimmen, welche verschiedenen Möglichkeiten der kontextuellen Bezugnahme bestehen. Wichtig für unsere Überlegungen ist, daß das Dativ-Objekt und die Lokalangabe im Vorfeld von (92b) bzw. (d) spezielle Forderungen an den Kontext stellen, während die Mittelfeldauszeichnung dieser Elemente als Satztopiks in (92a) bzw. (c) keine kontextuellen Anforderungen nach sich zieht. Man macht sich leicht klar, daß letzteres auch für Scrambling gilt.

Im folgenden sind die Unterschiede zwischen den drei betrachteten Bewegungen nochmals aufgeführt ${ }^{37}$ :

\begin{tabular}{|c|c|c|c|}
\hline & Topik-Voranstellung & Scrambling & Bewegung ins Vorfeld \\
\hline (ii) Einfacher Trigger & $\checkmark$ & $*$ & \\
\hline $\begin{array}{c}\text { (iii) Bewegt spezielle } \\
\text { Genitive/ Dative }\end{array}$ & $\checkmark$ & $\checkmark$ & $\checkmark$ \\
\hline $\begin{array}{c}\text { (iv) mögl. in V-Pro- } \\
\text { jektion im Vorfeld }\end{array}$ & $*$ & $\checkmark$ & $*$ \\
\hline $\begin{array}{c}\text { (v) Iterierbar } \\
\text { (vi) Bewegte Phrase ist } \\
\text { transparent }\end{array}$ & $\checkmark$ & $\checkmark$ & $\checkmark$ \\
\hline $\begin{array}{c}\text { (vii) Induziert Barriere } \\
\text { (viii) Satz-gebunden }\end{array}$ & $*$ & $*$ & $*$ \\
\hline (ix) notw. Kontextbe- \\
zug der Phrase
\end{tabular}

35 Natürlich haben die Sätze auch Verwendungen, bei denen die Phrasen im Vorfeld den minimalen Fokus darstellen, d.h. bei denen diese Sätze z.B. Antworten auf entsprechende W-Fragen sind. Diese Vewendung spielt für unsere Überlegungen keine Rolle.

36 Für (93b) gilt dies natürlich nur unter Berücksichtigung der Überlegung, auf die Fn. 25 verweist.

37 Für die Eigenschaft (ii) bei 'Vorfeldbesetzung' siehe Abschnitt 9, die Eigenschaft (iv) ist für Vorfeldbesetzung nicht einschlägig. 


\section{Einige Schlußfolgerungen}

In diesem abschließenden Kapitel sollen einige Schlußfolgerungen aus der Matrix (94) gezogen werden.

Die Eigenschaft (ii) von (94) betrifft die Tatsache, daß die durch (6) charakterisierte Voranstellung im Mittelfeld genau die Elemente mit Topikstatus betrifft. Im Unterschied hierzu hat nach unseren Kriterien die Umstellung von Phrasen unterhalb der Satzadverbiale (Scrambling) nichts mit Topikalität zu tun. Da die Topik-Voranstellung an eine bestimmte Eigenschaft geknüpft ist, fügt sie sich in die heute bevorzugte Sichtweise von Bewegung, wonach Bewegung genau dann stattfindet, wenn eine Eigenschaft bzw. ein Merkmal lizenziert wird. Insbesondere Rizzi (1997) hat dafür argumentiert, daß auch pragmatische Eigenschaften durch Merkmale kodiert werden, die zu lizenzieren sind. Hiernach werden etwa fokale oder topikale Phrasen in die Spec-Positionen entsprechender funktionaler Projektionen bewegt (FocP bzw. TopP), wo die Lizenzierung stattfindet ('Fokus-' bzw. 'Topik-Kriterium').

Wenn wir uns dieser Überlegung anschließen, folgt aus (6), daß im Mittelfeld des deutschen Satzes direkt oberhalb der Grundposition der Satzadverbiale eine TopP bzw. eine Folge von TopPs anzusetzen ist. Zwei der im letzten Abschnitt genannten Eigenschaften geben der Annahme von TopPs im Mittelfeld zusätzlich eine gewisse empirische Plausibilität. Durch die Topik-Voranstellung können Elemente bewegt werden, die nicht zu scrambeln sind (Eigenschaft (iii)). Was immer der Grund für Scrambling sein mag, Topik-Voranstellung dient dazu, das Topik-Kriterium zu erfüllen. Dieser Bewegung können somit alle Phrasen unterzogen werden, die das Topik-Merkmal tragen können. Durch die Annahme von TopPs (und dem Topik-Kriterium) kann solchen prinzipiellen Unterschieden zwischen Topik-Voranstellung und Scrambling Rechnung getragen werden. Die zweite Eigenschaft, die durch das Postulat strukturell ausgezeichneter Topikpositionen leichter zu erfassen ist, ist die Eigenschaft (iv). Daß keine Topiks im komplexen Vorfeld auftreten können, kann dann durch die Annahme erfaßt werden, daß nur Segmente unterhalb der ausgezeichneten Topikprojektion Teil der verbalen Projektion im Vorfeld sein können.

Rizzi (1997) schlägt vor, die CP-Projektion der Satzstruktur durch die folgende Kaskade funktionaler Projektionen zu ersetzen:

$$
\text { ForceP TopP* FocP TopP* FinP }
$$

Die verschiedenen Projektionen, die die A'-Projektion CP ersetzen, sind nach Rizzi ebenfalls A'-Projektionen. Da die Projektionen in (95) als Auffächerung der C-Domäne gedacht sind, stellt sich in Anbetracht unserer Beobachtungen unmittelbar das folgende Problem. Unter der C-Domäne eines deutschen Satzes versteht man gemeinhin den Bereich, der das Vorfeld und die linke Satzklammer umfaßt. Bereiche des Mittelfeldes wurden bislang nicht darunter subsumiert, so daß sich die durch (6) bestimmte Domäne nicht unmittelbar einer Projektion von (95) zuordnen läßt.

Da aber topikale Phrasen auch im Vorfeld auftreten können, könnte man versucht sein vorzuschlagen, daß die Kaskade funktionaler Projektionen in (95) nicht nur das Vorfeld 'abdeckt', sondern in das Mittelfeld 'hineinreicht'. Der von (6) identifizierte Topikbereich wäre unter dieser Annahme durch TopPs im Sinne von (95) aufgespannt. Ein Topik im Vorfeld stünde ebenfalls im Spec einer TopP. Der Unterschied zwischen 'äußerem Top' (d.h. einer Top-Projektion, die das Vorfeld konstituiert) und 'innerem Top' (d.h. einer Top-Projektion, die den Topikbereich des Mittelfeldes mitkonstituiert) wäre lediglich das Resultat der Oberflächenposition des finiten Verbs: 
Es ist jedoch unmittelbar deutlich, daß dieser Ansatz nicht durchführbar ist. Dies ergibt sich insbesondere aus dem im letzten Abschnitt angeführten Unterschied zwischen der Vorfeldbesetzung und der Topik-Voranstellung bezüglich Lokalität (Eigenschaft (viii)). Wenn 'äußeres Top' identisch wäre mit 'innerem Top', könnte nicht erklärt werden, warum ein 'inneres Top' nur von Phrasen desselben Satzes angesteuert werden kann, während ein 'äußeres Top' auch mögliche Zielposition von Elementen aus eingebetteten Sätzen ist. Desweiteren induziert ein 'inneres Top' keine Barriere für andere Mittelfeldtopiks oder für die Bewegung ins Vorfeld, während, wenn man Verbzweit-Komplementsätze annimmt (s. jedoch Fn. 34), zu erfassen ist, daß ein 'äußeres Top' jede weitere Bewegung blockiert.

Die Eigenschaft (ix) aus Abschnitt 8 zeigt jedoch, daß sich 'Vorfeldtopiks' und 'Mittelfeldtopiks' nicht nur in ihren syntaktischen Eigenschaften unterscheiden. Die Daten (92b, d) zeigen, daß Topiks im Vorfeld eine Diskursabhängigheit aufweisen, die bei Mittelfeldtopiks nicht zu finden ist. Diese Eigenschaft der 'Vorfeldtopiks' erinnert an Vallduvís 'link'-Konzept (vgl. Abschnitt 7), welches wir für 'Mittelfeldtopiks' zurückgewiesen haben. In einem Theorierahmen, welcher pragmatische Eigenschaften wie Topikalität, fokaler Status oder Diskursgebundenheit in der Syntax kodieren will, sollten daher 'Vorfeldtopiks' nicht in derselben funktionalen Projektion 'geprüft' werden wie 'Mittelfeldtopiks'.

Ich werde daher nicht dem Vorschlag Rizzis in (95) folgen, sondern an der traditionellen Vorstellung festhalten, daß die Vorfeldposition des deutschen Satzes durch die Spec-Position von CP konstituiert wird. Ebenfalls der Tradition folgend nehme ich an, daß der C-Kopf mit unterschiedlichen Merkmalen ausgestattet sein kann. Für alle (nichtdefektiven) Merkmale, die in einem C-Kopf auftreten können, gilt, daß sie eine Konstituente mit demselben Merkmal im Vorfeld lizenzieren. Zu diesen Merkmalen gehören z.B. das wh-Merkmal für Fragesätze und das Fokus-Merkmal für Sätze mit vorangestelltem fokalem Element. Desweiteren soll das 'link'-Merkmal dazugehören. Dies dient da$\mathrm{zu}$, die Vorfeldbesetzung in Sätzen wie (92b) und (d) zu lizenzieren. Die Phrasen im Vorfeld dieser Beispiele stellen an den Kontext eine 'familiarity'-Bedingung. Die durch das 'link'-Merkmal lizenzierten 'familiarity'-Topiks sind zu unterscheiden von den durch (6) ausgezeichneten 'aboutness'-Topiks des Mittelfeldes. Für diese sollen TopPs angesetzt werden.

Das Bild wird dadurch komplizierter, daß bekanntermaßen nicht jede Vorfeldbesetzung zu kontextuellen Anforderungen führt. Betrachten wir zunächst die folgenden Beispiele, die Lesarten mit vollkommen unmarkierter Vorfeldbesetzung aufweisen:

(97) a. Keiner konnte die Aufgabe lösen

b. Hans schaut sich jedes Fußballspiel an

c. Einem Mitbewohner wurde im Park die Geldbörse entwendet

d. Vor zwei Tagen konnte Paul das Problem lösen

e. Im Mittelalter haben Mönche viel Bier getrunken

Die Sätze in (97) sind neutral, insbesondere sind sie kontextuell neutral. Sie stellen keine Forderungen nach einem Bezug auf Gegebenes. Dabei kann die Phrase im Vorfeld ein 'aboutness'-Topik sein. (97b) z.B. kann so verstanden werden, daß Hans als Topik aufgefaßt wird. In (97) befinden sich Subjekte transitiver Verben, das Dativ-Objekt einer Passivkonstruktion, ein Temporaladverbial bzw. ein sog. rahmensetzendes Adverbial im Vorfeld. 
Die folgenden Sätze sind zwar nicht unmarkiert, aber auch hier stellen die Vorfeldelemente nicht notwendigerweise kontextuelle Anforderungen:

(98) a. Einigen Rentnern hat angeblich die Polizei Handschellen angelegt

b. Im Eisbärbecken hat erstaunlicherweise ein Zoobesucher ein Bad genommen

Betrachten wir zunächst die Daten in (97). Gisbert Fanselow (p.M.) hat vorgeschlagen, Sätze dieser Art als Instanz von sog. 'stylistic fronting' zu analysieren. Mit diesem Namen wird eine Voranstellungskonstruktion im Isländischen und Färöischen bezeichnet, bei der in Sätzen, die kein Satz-internes Subjekt aufweisen, eine andere Kategorie in die Subjekt-Position bewegt wird (vgl. Holmberg (2000)). Eine wichtige Bedingung für 'stylistic fronting' ist, daß jeweils nur jenes Satzglied des gleichen Satzes in die leere Subjektposition bewegt werden kann, welches von der Subjektposition minimal kkommandiert wird. Nach Holmberg (2000) dient 'stylistic fronting' lediglich dazu, die Forderung der fraglichen Spezifikator-Position nach Füllung mit phonetischem Material zu erfüllen. Homberg zeigt, daß 'stylistic fronting' in bestimmten Konstruktionen mit der Füllung der Position durch ein Expletiv alterniert.

Es kann hier nicht der Versuch gemacht werden, den Vorschlag Fanselows auszuarbeiten. Aber auffallend ist, daß sich die Sätze in (97) gerade dadurch auszeichnen, daß in ihnen jeweils jene Phrase im Vorfeld steht, die die höchste Mittelfeldposition aufweisen würde. Dies gilt in $(97 \mathrm{a}, \mathrm{b})$ für die transitiven Subjekte. Im Passivsatz (97c) hingegen hat das Dativobjekt eine höhere Basisposition als das Lokaladverbial und als das Subjekt. Ein Temporaladverbial wie in (97d) oder ein rahmensetzendes Adverbial wie in (97e) haben nach Frey \& Pittner (1998) eine höhere Basisposition als ein transitives Subjekt.

Die Vorfeldphrasen in (97b-e) können jedoch auch als Topiks aufgefaßt werden. Man beachte aber, daß sie auch dann das Vorfeld durch 'stylistic fronting' erreichen können. Sie haben als Topiks vor der Vorfeldplazierung die höchste Topikposition des Mittelfeldes eingenommen.

Die Annahme, daß die Sätze in (97) in der unmarkierten Lesart durch 'stylistic fronting' des am höchsten positionierten Mittelfeldelementes entstehen, erlaubt die folgende Vorhersage für das Subjekt in (97a). Dieses ist nicht topikfähig. Wenn es durch 'stylistic fronting' in die Vorfeldposition gelangt ist, dann nicht aus einer Topikposition. Daraus folgt, das kein 'stylistic fronting' vorliegen kann, wenn ein Satzadverbial oder gar ein Satztopik auftritt, da diese dem Vorfeld näher sind als eine Mittelfeldposition von 'keiner' ${ }^{38}$ :

(99) a. Keiner konnte bedauerlicherweise die Aufgabe lösen

b. Keiner konnte die Aufgabe bedauerlicherweise lösen

Im Unterschied zu (97a) besteht bei diesen Beispielen die Tendenz, das Subjekt im Vorfeld zu betonen. Einher geht damit eine fokusierte Lesart des Subjekts. In (99a) kann wegen dem Satzadverbial kein 'stylistic fronting' vorliegen, in (99b) wird dies darüber hinaus von dem topikalen Objekt verhindert.

Betrachten wir nun die Daten in (98). Diese Beispiele weisen eine nicht-kanonische Reihenfolge der Satzglieder auf. In (98a) geht ein Objekt einem Satzadverbial und dem transitivem Subjekt voraus. In (98b) trifft dies auf ein Lokaladverbial zu, welches nach Frey \& Pittner (1998) seine Grundposition unterhalb der Grundposition des transitiven

38 Nur unter I-contor oder Kontrastfokus könnte 'keiner' in den folgenden Beispielen zugrundeliegend eine Spitzenstellung im Mittelfeld einnehmen (vgl. (VIII) von Abschnitt 3.). 
Subjekts hat. Die nicht-kanonische Reihenfolge führt dazu, daß die Phrasen im Vorfeld eine hohe Salienz aufweisen. Man beachte aber, daß sich die gesteigerte Salienz gleichermaßen ergibt, wenn die fraglichen Phrasen im Topikbereich am Beginn des Mittelfeldes auftreten:

(100) a. da einigen Rentnern angeblich die Polizei Handschellen angelegt hat

b. da im Eisbärbecken erstaunlicherweise ein Zoobesucher ein Bad genommen hat

Diese Beobachtung legt nahe, daß auch für die Sätze in (98) eine Ableitung besteht, bei der die strukturell höchste Phrase des Mittelfeldes durch 'stylistic fronting' in das Vorfeld angehoben wird.

Damit ergibt sich das folgende Bild. Die Spezifikatorposition der CP eines deutschen Verbzweit-Satzes muß durch eine Konstituente besetzt werden. Dies kann auf unterschiedliche Weise geschehen. Eine erste Möglichkeit ist die Einsetzung eines Expletivs in diese Position. Eine andere Möglichkeit ist, die Position durch 'stylistic fronting' mit dem höchsten Mittelfeldelement zu besetzen. Nach Holmberg (2000) dienen die Einsetzung eines Expletivs und 'stylistic fronting' lediglich dazu, die Forderung nach phonologischem Material in der fraglichen Position zu erfüllen. Es wird durch diese Operationen kein zusätzlicher semantischer bzw. pragmatischer Effekt erzielt. Dies ist anders bei der dritten Möglichkeit, die Vorfeldposition zu füllen. In diesem Fall ist der C-Kopf mit semantisch/pragmatisch $\mathrm{zu}$ interpretierenden Merkmalen ([wh], [fok] oder [link]) ausgestattet. Es ist somit nur diese letzte Art der Vorfeldbesetzung, bei der qua Vorfeldbesetzung spezifische semantische oder pragmatische Effekte erzielt werden. ${ }^{39}$ Unter dieser Art der Vorfeldbesetzung ist für uns im folgenden jene relevant, die einen 'link' betrifft. Sind diese Behauptungen empirisch zu überprüfen? Schließlich haben die Sätze in (98) auch dann eine spezifische pragmatische Eigenschaft, wenn sie auf eine 'zugrundeliegende' Mittelfeldserialisierung (vgl. (100)) zurückgeführt werden. Man beachte jedoch, daß diese pragmatische Eigenschaft verschieden ist von der eines 'links'. Während es sich in (98) (wie in (100)) um die Salienz der strukturell höchsten Phrase handelt, geht es bei der Vorfeldbesetzung durch einen 'link' um Diskursangebundenheit.

'Stylistic fronting' ist eine Operation, die nur eine Phrase desselben Satzes betreffen kann. Die Vorfeldbesetzung durch einen 'link' hingegen ist nicht Satz-gebunden. Sie kann eine Phrase desselben Satzes betreffen, aber sie muß es nicht. Betrachten wir hierzu das folgende Beispielpaar:

(101) Erstaunliches ist passiert!

a. Das neue Buch von Rizzi hat Hans Maria zu lesen empfohlen

b. \#Das neue Buch von Rizzi hat Hans Maria zu lesen veranlaßt

39 Auch Pronomen können'stylistic fronting' unterzogen werden. Dies ist in (ib) für ein Objektpronomen illustriert:

(i) a. Da ihn Maria lange nicht mehr gesehen hat (wird sie den Hans heute anrufen)

b. Ihn hat Maria lange nicht mehr gesehen. (Deshalb wird sie den Hans heute anrufen)

Allerdings sind entsprechende Beispiele mit einem Objekt-es nicht möglich. Ein solches kann nicht durch 'stylistic fronting' ins Vorfeld gelangen. Ein Objekt-es im Vorfeld scheint stets ein 'link' zu sein, so auch im folgenden Beispiel aus Gärtner \& Steinbach (2000):

(ii) $[\mathrm{A}:] \quad$ Wie ist denn das Kind zu dem Buch gekommen?

[B:] Es hat ihm jemand geschenkt 
Die Sätze (101a) und (b) unterscheiden sich in ihrer Angemessenheit im gegebenen Kontext. Dieser pragmatische Unterschied kann mit einem syntaktischen Unterschied der beiden Sätze erklärt werden. Die Infinitivkonstruktion in (101a) erlaubt, kohärent konstruiert zu werden, der Infinitiv in (101b) hingegen ist obligatorisch inkohärent (s. z.B. Haider (1993)). Daraus ergibt sich der folgende Unterscheid:

(102) a. da das neue Buch von Rizzi Hans Maria zu lesen empfohlen hat

b. *da das neue Buch von Rizzi Hans Maria zu lesen veranlaßt hat

In der kohärenten Konstruktion ist die Voranstellung des Infinitiv-Objekts vor das Matrix-Subjekt möglich, nicht aber in der inkoränten. Die kohärente Konstruktion verhält sich wie eine monosententiale Struktur. Aus diesem Grund ergibt sich die Möglichkeit, (101a) aus (102a) via 'stylistic fronting' abzuleiten. Eine entsprechende Option besteht für (101b) nicht. Da die Vorfeldphrase in (101b) nicht durch 'stylistic fronting' vorangestellt wurde, muß sie ein 'link' sein, d.h. sie verlangt eine Anbindung an den Diskurs. Diese ist im Kontext von (101) nicht gegeben, daher ist der Satz nicht angemessen.

Wenn der Kontext den Anforderung eins 'links' genügt, sind beide Sätze möglich: Was ist mit dem neuen Buch von Rizzi?

a. Das neue Buch von Rizzi hat Hans Maria zu lesen empfohlen

b. Das neue Buch von Rizzi hat Hans Maria zu lesen veranlaßt

Der Unterschied zwischen (101a) und (101b) belegt somit den pragmatischen Unterschied zwischen der Vorfeldbesetzung durch ein Mittelfeldtopik mittels 'stylistic fronting' und einer Vorfeldbesetzung, welche an das 'link'-Merkmal des C-Kopfes gebunden ist.

Abschließend sollen die syntaktischen Eigenschaften, die in (94) aufgeführt sind, kurz kommentiert werden. Die Bemerkungen haben allerdings einen vorläufigen Charakter, da hier keine Theorie der Bewegungen entwickelt werden kann. Vergleichen wir zunächst Topik-Voranstellung und Vorfeldbesetzung. Im aktuellen Model der ChomskyGrammatik (Chomsky (1999)) wird sukzessiv-zyklische Bewegung durch (defektive) Merkmale in den 'Zwischenpositionen' ermöglicht, welche zwar von der bewegten Phrase getilgt werden, welche aber die bewegte Phrase nicht lizenzieren. Der Unterschied bezüglich Lokalität in (94viii) wäre daher wie folgt zu erfassen. C kann mit einem uninterpretierbarem und defektivem Merkmal $P_{\text {link }}$ ausgestattet sein, welches die sukzessivzyklische Bewegung einer Phrase mit dem 'link'-Merkmal ermöglicht. In seiner Zielposition wird das 'link'-Merkmal der bewegten Phrase durch ein 'link'-Merkmal des Kopfes lizenziert. Für das Satz-interne Topik sind keine entsprechenden Merkmale in C vorhanden. Ein 'aboutness'-Topik kann daher seinen Satz nicht verlassen. Wenn ein Satzinternes Topik im Vorfeld auftritt, dann nur via 'stylistic fronting'. 'Stylistic fronting' dient lediglich dazu, die Forderung nach lexikalischer Füllung von [Spec, CP] eines Verbzweit-Satzes zu erfüllen. Letzteres wird codiert durch ein 'EPP'-Merkmal [phonologisch], wodurch die lexikalische Füllung der Spec-Position erzwungen wird (s. Holmberg $(2000)){ }^{40}$

Nach Rizzi (2000) induzieren TopPs weder für Phrasen mit einem anderen Merkmal noch für Phrasen, welche ebenfalls das Topik-Merkmal tragen, einen Minimalitätseffekt. Dies entspricht unseren Beobachtungen. Auf der anderen Seite verhindert jede Art von

40 Die anderen C-Merkmale [wh], [fok], [link] oder $P_{\text {link }}$ wären im System von Chomsky (1999) auch mit einem EPP-Merkmal zu assoziieren, das die Füllung der Spec-Position erzwingt. 
Vorfeldbesetzung die zyklische Bewegung, da ein C-Kopf nicht mehr als ein EPPMerkmal hat. Dies ergibt (94vii). Für (94vi) gibt es allerdings meines Wissens beim momentanen Stand der Bewegungstheorie keine Erklärung. Dies aber ist unabhängig von unserer Fragestellung. Es ist ein altes Problem für die Standardtheorie, daß beliebige Umstellungen im Mittelfeld zu keinem 'freezing'-Effekt führen. Die Opakheit der Vorfeldphrase hingegen ist einer der klassischen CED-Effekte. ${ }^{41}$ Bezüglich der Eigenschaft (94v) ist zu bemerken, daß die Iterierbarkeit der Topik-Voranstellung der Annahme Rizzis in (95) entspricht, daß TopPs rekursiv sein können. CP ist nicht rekursiv im Deutschen, daher kann es keine mehrmalige Vorfeldbesetzung geben.

Betrachten wir schließlich die Eigenschaften (ii) und (iii). Bezüglich (ii) können wir nach den obigen Überlegungen feststellen, daß die für unsere Diskussion relevanten Vorfeldbesetzungen ebenfalls durch eindeutige Trigger ausgelöst werden. Dies ist zum einen das pragmatisch zu interpretierende 'link'-Merkmal, zum anderen das Merkmal [phonologisch], das durch 'stylistic fronting' oder mittels eines Expletivs erfüllt wird. Die Eigenschaft (iii) ist zum jetzigen Zeitpunkt schwer zu interpretieren, da nicht bekannt ist, warum Verb-nahe Dative und Genitive gegen Scrambling resistent sind. Um letzteres zu verstehen, müßte über die Funktion von Scrambling mehr bekannt sein. Aber es erscheint natürlich, daß sich Topik-Voranstellung und die Vorfeldbesetzung bezüglich (iii) gleich verhalten, da die Auszeichnung als 'aboutness'-Topik und die Auszeichnung als 'link' ('familiarity'-Topik) verwandte Eigenschaften betreffen.

Betrachten wir abschließend zwei Eigenschaften von (94) bezüglich TopikVoranstellung und Scrambling. Unter (vi) wurde vermerkt, daß bei beiden Operationen die bewegte Phrase transparent ist für die Extraktion einer Subkonstituente. Oben wurde bereits erwähnt, daß es für die Standardtheorie ein Problem darstellt, daß Scrambling im deutschen Mittelfeld zu keinem 'freezing'-Effekt führt. Haider \& Rosengren (1998) entwickeln daher eine Analyse von Scrambling, welche keine funktionalen Projektionen 'ansteuert'. Scrambling ist vielmehr eine Bewegung, die eine Phrase an die V-Projektionslinie adjungiert. Da sich die 'gescrambelte' Phrase nicht im Spec einer funktionalen Projektion befindet sondern in einer lexikalisch regierten Position, erwartet man keinen 'freezing'-Effekt.

Daß die Topik-Voranstellung ebenfalls keinen 'freezing'-Effekt zeigt, könnte als Evidenz dafür angesehen werden, daß der Topikbereich des Mittelfeldes nicht durch funktionale Top-Projektionen konstituiert wird. Die Topik-Voranstellung wäre ebenfalls Adjunktion an eine V-Projektion. Die Satzadverbiale würden ausschließlich für die Semantik/Pragmatik-Komponente das Mittelfeld in einen Topikbereich und einen KommentarBereich aufteilen.

Die Erklärung der Tatsache, daß im komplexen Vorfeld kein Topik auftreten kann $((94 i v))$, wird in einem solchen Rahmen der Semantik/Pragmatik überlassen. Aber auch in einem solchen Ansatz müssen, um den Befund (94iii) zu erfassen, Phrasen mit pragmatisch zu interpretierenden Merkmalen ausgestattet sein. Denn die Scramblingresistenten Phrasen dürfen nur bewegt werden, wenn sie topikal sind. Da die Zielpositionen von Topiks und Nicht-Topiks strukturell nicht unterschieden sind, muß zudem die Semantik/Pragmatik alle Strukturen aussondern, in denen die topikalen Phrasen keine zusammenhängende Domäne vor einem eventuell vorkommenden Satzadverbial und den nicht-topikalen Phrasen bilden. Damit erhalten wir jedoch eine unplausible Charakterisierung der Verhältnisse im Mittelfeld. Ein Merkmal [topik] ist zwar für den Input der syntaktischen Regel 'Mittelfeldumstellung' relevant. Für die Überprüfung der Wohlge-

4! Man beachte jedoch, daß die Beobachtungen (94vii) und (vi) bezüglich Vorfeldbewegung gegenstandslos sind, wenn man Reis (1997) folgt, s. Fn. 34. 
formtheit des Outputs spielt das Merkmal syntaktisch jedoch keine Rolle. Die Überprüfung des Outputs erfolgt vielmehr ausschließlich mittels eines semantisch/pragmatischen Filters. Insgesamt läßt sich somit festellen, daß für die Transparenz einer topikalen Phrase $((94 \mathrm{vi}))$ zum jetzigen Zeitpunkt keine befriedigende Erklärung vorliegt.

Sowohl Topik-Voranstellung als auch Scrambling sind Satz-gebunden (Eigenschaft (94viii)). Wir haben unter 'Scrambling' jene Umstellungen verstanden, die sich unterhalb der Topikbereichs vollziehen. Nach (VIII) in Abschnitt 3 befindet sich direkt oberhalb der Topikbereichs die Zielposition einer nicht Satz-gebundenen Bewegung unter I-Kontur oder Kontrastfokus. Wir stoßen somit auf eine Peripherieeigenschaft der Topiks. Die Topik-Voranstellung positioniert Phrasen in die linke Peripherie jener Domäne des deutschen Satzes, die durch lokale Bewegungen charakterisiert ist.

Es wurde häufig beobachtet, daß in verschiedenen Sprachen die Tendenz besteht, Topiks linksperipher auszuzeichnen. Auf das Deutsche bezogen, wurde 'linksperipher' häufig so aufgefaßt, daß damit das Vorfeld gemeint ist. Dies ist nach unseren Beobachtungen für 'aboutness'-Topiks nicht richtig. Aber es ist richtig, daß die Topik-Auszeichnung auch im Deutschen linksperipher erfolgt. 'Aboutness'-Topiks werden linkssperipher in der Domäne lokaler Umstellungsoptionen ausgezeichnet. Die Auszeichnung eines 'links' hingegen erfolgt im Vorfeld, also in der linken Peripherie jener Domäne, die syntaktisch durch nicht-lokale Umstellungsoptionen charakterisiert ist.

\section{Literatur}

Brand, M., M. Reis, I. Rosengren \& I. Zimmermann (1992): Satztyp, Satzmodus und Illokution. In: I. Rosengren (ed.): Satz und Illokution, Bd. 1, Tübingen: Niemeyer, 1-90.

Büring, D. (1995): The 59th Street Bridge Accent. Phil. Diss. Universität Tübingen.

Carlson, G. (1978): Reference to Kinds in English. New York: Garland.

Chomsky, N. (1999): Derivation by Phase. Ms., MIT.

Diesing, M. (1992): Indefinites. Cambridge, Mass.: MIT Press.

Diesing, M. (1997): Yiddish VP Order and the Typology of Object Movement in Germanic. In: Natural Language and Linguistic Theory, 15, 369-427.

Drubig, B. (1992): Zur Frage der grammatischen Repräsentation thetischer und kategorischer Sätze. In: J. Jacobs (ed.): Informationsstruktur und Grammatik. Linguistische Berichte, Sonderheft 4/1991-92, 142-195.

Erteschik-Shir, N. (1997): The Dynamics of Focus Structure. Cambridge: University Press.

Frey, W. \& K. Pittner (1998): Zur Positionierung der Adverbiale im deutschen Mittelfeld. Linguistische Berichte 176, 489-534.

Gärtner, H.-M. \& M. Steinbach (2000): Conditions on the Scrambling and Fronting of Reduced Pronominals in Dutch and German. Ms., Univ. Potsdam, Univ. Mainz.

Grundzüge einer deutschen Grammatik (1981): Von einem Autorenkollektiv unter der Leitung von KarlErich Heidolph, Walter Flämig und Wolfgang Motsch. Berlin: Akademie-Verlag.

Haftka, B. (1995): Syntactic Positions for Topic and Contrastive Focus in the German Middlefield. In: I. Kohlhof, S. Winkler \& H.B. Drubig (eds): Proceedings of the Goettingen Focus Workshop. Arbeitspapiere des SFB 340, Bericht Nr. 69, 137-157.

Haider (1993): Deutsche Syntax - Generativ. Tübingen: Narr.

Haider, H. \& I. Rosengren (1998): Scrambling. Sprache und Pragmatik 49, Lund.

Halliday, M. (1967): Intonation and Grammar. Den Haag: Mouton.

Hetland, J. (1992): Satzadverbien im Fokus. Tübingen: Narr.

Hockett, Ch. F. (1958): A Course in Modern Linguistics. New York: McMillan.

Holmberg, A. (2000): Scandinavian Stylistic Fronting: How Any Category Can Become an Expletive. In: Linguistic Inquiry, 31, 445-483.

Jacobs, J. (1984): Funktionale Satzperspektive und Illokutionssemantik. In: Linguistische Berichte 91, 25 58.

Jacobs, J. (1996): Bemerkungen zur I-Topikalisierung. Sprache und Pragmatik, Arbeitsbericht. Universität Lund. 


\section{Werner Frey}

Jacobs, J. (1999): The Dimensions of Topic-Comment. Draft, Universität Wuppertal.

Jäger, G. (1996): Topics in Dynamic Semantics. Phil. Diss. Berlin, Humboldt-Universität.

Jäger, G. (1999): Topic, Focus, and Weak Quantifiers. In: P. Bosch \& R. v. der Sandt (eds.): Focus: Linguistic, Cognitive, and Computational Perspectives. Cambridge: Cambridge University Press, 187. 212.

Jäger, G. (1999a): Stage Levels, States, and the Semantics of the Copula. In: E. Lang \& L. Geist (eds.): Kopula-Prädikativ-Konstruktionen als Syntax/Semantik-Schnittstelle (= ZAS Papers in Linguistics 14), 65-94.

Jörke, V. (1997): Zur Rolle des Subjekts in der Informationsstruktur: Die Distinktion 'thetisch-kategorisch'. Magisterarbeit, Universität Stuttgart.

É. Kiss, K. (1995): Introduction. In: K. É. Kiss (ed.): Discourse Configurational Languages. Oxford: Oxford University Press, 3-27.

É. Kiss, K. (1995a): NP Movement, Operator Movement, and Scrambling in Hungarian. In: K. É. Kiss (ed.): Discourse Configurational Languages. Oxford: Oxford University Press, 207-243.

Kornfilt, J. (1997): Turkish. London: Routledge.

Krifka, M. (1992): A Compositional Semantics for Multiple Focus Constructions. In: J. Jacobs (ed.): Informationsstruktur und Grammatik. Linguistische Berichte, Sonderheft 4/1991-92, 17-53.

Kuno, S. (1972): Functional Sentence Perspective: A Case Study from Japanese and English. In: Linguistic Inquiry, 3, 269-336.

Laenzlinger, C. (1998): Adverbs, Pronouns, and Clause Structure in Romance and Germanic. Amsterdam: Benjamins.

Lambrecht, K. (1994): Information Structure and Sentence Form. Cambridge: Cambridge University Press.

Lasnik, H. \& M. Saito (1992): Move $\alpha$ : Conditions on Its Application and Output. Cambridge, Mass: MIT Press.

Lenerz, J. (1993): Zur Syntax und Semantik deutscher Personalpronomina. In: M. Reis (ed.): Wortstellung und Informationsstruktur. Tübingen: Niemeyer (Linguistische Arbeiten, 306), 117-153.

Maienborn, C. (1998): The Grammar and Pragmatics of Locative Modifiers. Ms., HU Berlin.

Meinunger, A. (2000):. Syntactic Aspects of Topic and Comment. Amsterdam: Benjamins.

Molnár, V. (1991): Das TOPIK im Deutschen und Ungarischen. Stockholm (Lunder germanistische Forschungen 58).

Molnár, V. (1998): Topic in Focus - On the Syntax, Phonology, Semantics and Pragmatics of the So-Called 'Contrastive Topic' in Hungarian and German. In: Acta Linguistica Hungarica, Vol 45 (1-2), 89 166.

Müller, G. \& W. Sternefeld (1993): Improper Movement \& Unambiguous Binding. Linguistic Inquiry 24, 461-507.

Pittner, K. (1999): Adverbiale im Deutschen. Tübingen: Staufenberg.

Reinhart, T. (1981): 'Pragmatics and Linguistics: An Analyssis of Sentence Topics'. Philosophica 27, 53 94.

Reinhart, T. (1995): Interface Strategies. OTS Working Papers, Utrecht University.

Reis, M. (1997): Zum syntaktischen Status unselbständiger Verbzweit-Sätze. In: C. Dürscheid, K.H. Ramers \& M. Schwarz (eds.): Sprache im Fokus: Festschrift für Heinz Vater zum 65. Geburtstag. Tübingen, Niemeyer, 121-144.

Rizzi, L. (1997): The Fine Structure of the Left Periphery. In: L. Haegeman (ed.): Elements of Grammar. Handbook in Generative Syntax. Dordrecht: Kluwer, 281-337.

Rizzi, L. (2000): Locality and Left Periphery. Vortrag, Conference on 'The Interaction between Syntax and Pragmatics', University College London, April 2000.

Schwarzschild, R. (1999): Givenness, Avoid F and Other Constraints on the Placement of Accent. In: Natural Language Semantics 7, 144-177.

Vallduví , E. (1992): The Informational Component. New York, Garland.

Vallduví, E. \& E. Engdahl (1996): The linguistic realization of information packaging. In: Linguistics 34, 459-519.

Zimmermann, I. (1999): Die Integration topikalischer DPs in die syntaktische und semantische Struktur von Sätzen. In: M. Doherty (ed.): Sprachspezifische Aspekte der Informationsverteilung. Berlin: Akademie-Verlag, (= Studia Grammatica 47), 41-60. 